\title{
TRIFOLIUM SECT. TRIFOLIUM. II. ESTUDIO CARIOLÓGICO
}

\author{
Adolfo MUÑOZ RODRÍGUEZ
}

\begin{abstract}
RESUMEN. Trifolium sect. Trifolium. II. Estudio cariológico. Se estudian desde el punto de vista cariológico las especies peninsulares del género Trifolium pertenecientes a la sect. Trifolium. Se aporta el número cromosómico y la morfología de los cromosomas, tamaño y asimetría, para 25 especies, y se discuten los resultado en base a la sistemática, ciclo de vida y distribución, teniendo en cuenta la bibliografía consultada. Para las especies estudiadas se comparan los índices de asimetría de Stebbins y Romero.
\end{abstract}

Palabras clave. Trifolium, Leguminosae, cariología.

SUMMARY. Trifolium sect. Trifolium. II. Karyological study. Trifolium species belonging sect. Trifolium spread in the Iberian Peninsula are studied from the cytological point of view. Chromosome number and morphology, size and asimmetry for 25 species are given, and the results are discussed in regard to systematics, life cycle and distribution, taking into account the consulted literature. Stebbins and Romero asimmetry indices are compared for the studied species.

Key words. Trifolium, Leguminosae, karyology.

\section{INTRODUCCIÓN}

Dentro del ámbito de la sect. Trifolium, además de mencionar a algunos autores clásicos en los estudios cariológicos del género como son Bleier (1925 a y b), Karpechenko (1925) y Wexelsen (1928) entre otros, se destacan las contribuciones de Larsen (1960), el cual fue el primer autor en detectar una especie con número diploide $2 \mathrm{n}=10$ (T. scabrum), para la que Karpechenko (1925) había señalado previamente $2 \mathrm{n}=16$; y Britten (1963), quién recopila la información cariológica publicada hasta esa fecha correspondiente al género Trifolium, y estima en 19 las especies de la sect. Trifolium de las que se conocía el número cromosómico.
Los primeros cariotipos los da a conocer Pritchard (1967) para cuatro especies de la sección Trifolium, y en esta misma línea publica más tarde una nueva aportación en la que estudia 11 especies de la sección (Pritchard, 1969), 5 de las cuales no poseen precedentes cariológicos. Posteriormente, Anderson et al. (1972) establecen el cariotipo de 3 especies de la sect. Trifolium. Por último, deben mencionarse los trabajos sobre la flora búlgara de Petrova \& Kozuharov (1982 a y b) quienes aportan datos, no sólo sobre el número cromosómico, sino también sobre la morfología de los cromosomas de 30 especies de esta sección.

En la Península Ibérica destacan los trabajos de Angulo et al. (1969, 1970, 1971, 1972 
a y b) y González-Bernáldez et al. (1973), quienes estudian los cariotipos de 18 especies en su mayoría peninsulares (12 de ellas pertenecientes a la sect. Trifolium). Y en lo concerniente a la flora portuguesa, destacar los trabajos de Fernandes y Santos (1971 y 1975), Fernandes'et al. (1977) y Fernandes y Queiros (1978), que han aportado información cariológica sobre 19 especies de la sección, para las que efectúan además comentarios de interés acerca de su morfología cromosómica.

La sect. Trifolum está representada en la Península Ibérica e Islas Baleares por un total de 34 táxones pertenecientes a 27 especies (Muñoz, 1992 b), siendo la sección del género mejor representada en dicha área. A ella pertenecen especies de alto valor pratense como son $T$. pratense L., $T$. incarnatum L. y $T$. alexandrinum L. En el presente trabajo se estudia el número cromosómico de 27 táxones pertenecientes a 25 de las especies peninsulares, aportánse datos acerca de la morfología cromosómica para 24 de éstas. Para las 2 especies de las que no se dispuso de material, T. phleoides Pourret ex Willd. y T. pannonicum Jacq., se recopilan los datos encontrados en la bibliografía consultada.

\section{MATERIAL Y MÉTODOS}

La mayor parte del material utilizado para el estudio cariológico proviene de plantas crecidas a partir de semillas recolectadas durante el año 1986, utilizándose en algún caso semillas procedentes de pliegos conservados en el herbario de la Facultad de Biología de Sevilla (SEV). Las semillas de $T$. incarnatum y $T$. alexandrinum fueron suministradas respectivamente, por el Centro de Biología Experimental del Consejo Superior de Investigaciones Científicas de Jaca (Huesca), y por el Instituto Botánico «Julio Henriques» de la Universidad de Coimbra.

Todas las observaciones se han realizado sobre mitosis, en radículas obtenidas entre el primer y quinto día a partir de la germinación de las semillas, por considerarse un material óptimo debido al elevado número de placas mitóticas. La germinación fue favorecida con un pretratamiento de 30 minutos con ácido sulfúrico concentrado (98\%), actuando como abrasivo (Small \& Joffe, 1967; Muñoz, 1992 a), salvo cuando no se dispuso de un número elevado de semillas, que se procedió directamente a la escarificación manual, provocando incisiones en la testa con una cuchilla.

Las plántulas fueron incluidas en 8hidroxiquinoleína 0'002 M (Tjio \& Levan, 1950), durante un periodo de tiempo que osciló entre tres y cuatro horas y media, período durante el cual las muestras se mantenían a 4 ${ }^{\circ} \mathrm{C}$. Posteriormente se lavaban con agua destilada y se fijaban en alcohol absoluto-ácido acético (3:1), también a $4^{\circ} \mathrm{C}$, donde permanecían un tiempo mínimo de 48 horas antes de la extracción de raices para su tinción y estudio.

La muestras fueron teñidas con carmín alcohólico-clorhídrico (Snow, 1963) durante 2-4 días a $35 \mathrm{C}^{\circ}$, al cabo de los cuales los extremos radiculares eran colocados sobre un portaobjetos inmersos en una gota de ácido acético (45\%), se aplastaban y se observaron al microscopio óptico.

De cada población se estudiaron por termino medio 5 radículas (de cinco individuos diferentes), tomándose datos relativos al número cromosómico y sobre la morfología de los cromosomas. De cada población se tomaron fotografías de las mejores placas metafásicas observadas (entre 1 y 5).

Las mediciones cromosómicas se efectuaron sobre placas metafásicas fotografiadas y ampliadas de 2400 a 6500 veces, no observándose en general para ninguna especie variaciones de tamaño interpoblacionales, por lo que el tamaño de los cromosomas dado en el presente estudio hace referencia siempre a la media calculada. En el caso de los satélites los datos biométricos son muy inexactos, debido a la gran dificultad que implica su medición, ya que como regla general, la constricción secundaria que los aisla del resto del cromosoma siempre se encuentra muy cercana al centrómero, por lo que es dificil de discernir este punto.

Para el tamaño de los cromosomas se ha seguido la terminología propuesta por Stebbins (1938), y para su morfología la de Levan et al. (1965). La asimetría cariotípica se ha calculado según los grados propuestos por Stebbins (1971), basados en la proporción existente entre la longitud del cromosoma más grande del cariotipo y el más pequeño, así como el porcentaje de cromosomas cuyo brazo más largo es el doble del brazo más corto. Igualmente, y en relación también con la asimetría cromosómica, se han calculado también los 
índices $\mathrm{A}_{1}$ y $\mathrm{A}_{2}$ establecidos por Romero (1986), el primero dependiente de la relación media entre el brazo largo y corto y el segundo, de la homogeneidad de sus tamaños.

\section{RESULTADOS}

\section{Subsect. Trifolium}

Trifolium pratense L. susbsp. pratense var. pratense

Material estudiado: GERONA. Enre Olot y Figueras, 10.VIII.86, A. Muñoz (UNEX 9899; 2n=14). GRANADA. Trevélez, 29.VIII.86, A.
Muñoz (UNEX 4790; 2n=14).

El número diploide encontrado $(2 n=14)$ coincide con los recuentos anteriores para este taxon (tab. 1), exceptuando a Krogulevich (1976), que halla $2 \mathrm{n}=16$, número dudoso si se considera la gran concordancia en resultados por parte del resto de los investigadores.

Wipf \& Cooper (1938) y Tatuno \& Kodama (1965) indican además niveles tetraploide y hexaploide en el taxon, muy frecuentes en individuos obtenidos por el hombre en experiencias de mejora forrajera. Con este fin, el uso de la colchicina es algo frecuente y bien conocido como método al uso en la obtención de tetraploides en el grupo (Levan, 1942 y 1945; Brewbaker, 1952; Bragdo, 1955; Evans, 1955; Povilaitis \& Boyes,

\begin{tabular}{|c|c|c|c|}
\hline & $\mathrm{n}$ & $2 n$ & Origen \\
\hline ANGULO et al. (1972 a y b, y 1981) & \multirow{7}{*}{7} & 14 & España \\
\hline ARUTIONOVA (1940) & & 14 & Rusia \\
\hline BLEIER (1925 a y b) & & 14 & Alemania \\
\hline BUTTERFASS (1960) & & 14 & Alemania \\
\hline CODIGNOLA \& MAFFEI (1981) & & 14 & Italia \\
\hline EL-BABA (1976) & & 14 & Grecia \\
\hline FERNANDES \& SANTOS (1971 y 1975) & & 14 & Portugal \\
\hline GADELLA \& KLIPHUIS (1966 y 1968 b) & \multirow{3}{*}{$\begin{array}{l}7 \\
7\end{array}$} & 14 & Holanda \\
\hline KARPECHENKO (1925) & & 14 & Rusia \\
\hline KAWAKAMI (1930) & & & S Europa \\
\hline KLIPHUIS \& WIEFFERING (1979) & \multirow{6}{*}{7} & 14 & Rusia \\
\hline KODAMA $(1967,1970)$ & & 14 & Japón \\
\hline KOZUHAROV et al. (1974) & & 14 & Bulgaria \\
\hline KROGULEVICH (1976) & & 16 & Rusia \\
\hline KUZMANOV \& STANCEV (1972) & & 14 & Bulgaria \\
\hline LÖVE \& LÖVE $(1944,1956)$ & & 14 & $\begin{array}{r}\text { Escandinavia e } \\
\text { Islandia }\end{array}$ \\
\hline MAJOVSKY et al. (1970 a, 1975 y 1978) & \multirow{4}{*}{$\begin{array}{r}7 \\
-7\end{array}$} & 14 & Checoslovaquia \\
\hline MORIYA \& KONDO (1950) & & & Japón \\
\hline MULLIGAN (1984) & & & Canadá \\
\hline NIELSEN (1975) & & 14 & Finlandia, Francia \\
\hline & & Alemania, $\mathrm{N}$ & a, Rumaníay Suiza \\
\hline NODA (1946) & \multirow{11}{*}{7} & 14 & Japón \\
\hline PETROVA \& KOZUHAROV (1982 a) & & 14 & Bulgaria \\
\hline PODLECH \& DIETERLE (1969) & & 14 & Afganistán \\
\hline POGAN et al. (1982) & & 14 & Polonia \\
\hline REESE (1953) & & 14 & Alemania \\
\hline SCHWER \& CLEVELAND (1972) & & 14 & Sin indicación \\
\hline TATUNO \& KODAMA $(1965) *$ & & $14,28,56$ & Japón \\
\hline TISCHLER (1934 y 1937) & & 14 & Alemania \\
\hline UOTILA \& PELLINEN (1985) & & 14 & Finlandia \\
\hline van LOON \& de JONG (1978) & & 14 & Francia \\
\hline van LOON \& KIEFT (1980) & & 14 & Yugoslavia \\
\hline WEXELSEN (1928) & \multirow[t]{3}{*}{7} & 14 & Estados Unidos \\
\hline WIPF (1939) & & 14 & África \\
\hline WIPF \& COOPER $(1938) *$ & & $14,28 *$ & Sin indicación \\
\hline
\end{tabular}

Tabla 1. Números cromosómicos en Trifolium pratense subsp. pratense. * recuentos efectuados en nódulos radicales. 
1956; Wexelsen \& Mikaelsen, 1958; y Turesson, 1962), así como el uso de $\mathrm{N}_{2} \mathrm{O}$ (Taylor et al., 1976 y 1980), así como el aprovechamiento de gametos $2 n$ originados de manera frecuente y espontánea en determinadas variedades (Parrot \& Smith, 1984; Parrot et al., 1985 y Tofte \& Smith, 1989). Los tetraploides así obtenidos poseen ciertas ventajas en su cultivo pero presentan numerosas irregularidades meióticas, que entrañan una abundante descendencia aneuploide que impide el buen establecimento de estas poblaciones (Ellerström \& Sjödin, 1966).

Los resultados obtenidos (tab. 11, fig. 1: A y B) muestran un cariotipo formado por cromosomas medianamente pequeños con ciertas diferencias de tamaño entre parejas, aunque no llegan -en las poblaciones estudiadas- al extremo señalado por Angulo et al. (1972 a) que indican que el par mayor es dos veces más grande que el menor. En las poblaciones estudiadas se evidencia la existencia de dos pares submetacéntricos, y no uno sólo como indicaron Angulo et al. (1972 a), observación que puede corroborarse también en la ilustración mostrada por Petrova \& Kozuharov (1982a). El par IV del cariograma está satelizado, abarcando los satélites el 4,5\% del tamaño total del complemento.

Trifolium pratense subsp. baeticum (Boiss.) C. Vicioso

Material estudiado: CÁDIZ. Ubrique, Garganta de la Barrida, 11.VII.86, A. Muñoz y J. M.Polo (UNEX 9913; $2 n=14$ ).

Para este taxon sólo se conocía previamente el recuento, coincidente con el efectuado aquí, de Valdés y González-Bernáldez (1972).
El cariotipo (tab. 11, fig. 1: C y D) es muy similar al de la subespecie pratense salvo en la ordenación de algunas parejas (lo que muy bien podría imputarse a la inexactitud de las mediciones).

Trifolium pallidum Waldst. \& Kit.

Material estudiado: CÁDIZ. Entre El Bosque y Ubrique, 10.VII.86, A. Muñoz y J. M. Polo (UNEX 4788; $2 n=16$ ). Entre Villamartín y Jerez de la Frontera, 11.VII.86, A. Muñoz y J. M. Polo (UNEX 4799; 2n=16).

El número hallado coincide con los encontrados por Pritchard (1969) en plantas griegas; Schwer \& Cleveland (1972), con material de origen desconocido, y Kozuharov et al. (1975) y Petrova \& Kozuharov (1982 a) en plantas búlgaras. Para la Península Ibérica se conoce el recuento efectuado por Valdés y González-Bernáldez (1972) en plantas del S de España.

En relación con las características cromosómicas sólo Petrova \& Kozuharov (1982 a) habían efectuado algunas observaciones, que en líneas generales coinciden con los datos expuestos aquí salvo en la diferente terminología (tab. 11, fig. 1: E y F).

Si se compara con las dos subespecies $T$. pratense, se comprueba que el índice $\mathrm{A}_{1}$ es similar en ambas especies, pero el índice $\mathrm{A}_{2}$ es algo mayor en $T$. pallidum.

\section{Subsect. Diffusa Muñoz}

\section{Trifolium diffusum Ehrh.}

Material estudiado: CÁCERES. Plasencia, 27.VI.86, J. A. Devesa, J. Herrera y A. Muñoz (UNEX 9906: 2n=16).

\begin{tabular}{|c|c|c|c|}
\hline . & $\mathrm{n}$ & $2 n$ & Origen \\
\hline BLEIER (1925 a y b) & $48-49$ & & Alemania \\
\hline CINCURA (1965) & & 80 & Checoslovaquia \\
\hline EVANS (1962) & & & Sin indicación \\
\hline FERNANDES \& QUEIROS (1978) & & 80 & Portugal \\
\hline HOLUB et al. (1972) & & $\begin{array}{c}64,79,80+0-2 B \\
82\end{array}$ & Checoslovaquia \\
\hline KARPECHENKO (1925) & & c. 80 & Rusia \\
\hline KOZUHAROV et al. (1972) & & 64 & Bulgaria \\
\hline LÖVE \& LÖVE (1944) & & c. 126 & Escandinavia \\
\hline LÖVE \& LÖVE (1961) & & c. 84 & C y N Europa \\
\hline \multirow{2}{*}{\multicolumn{4}{|c|}{ Yugoslavia, Checoslovaquia y Hungría }} \\
\hline & & & \\
\hline NILSSON \& LASSEN (1971) & & c.76 & Austria y \\
\hline PETROVA \& KOZUHAROV (1982 a) & & 64 & $\begin{array}{r}\text { ugoslavia } \\
\text { Bulgaria }\end{array}$ \\
\hline POGAN et al. (1982) & & 80 & Polonia \\
\hline TAYLOR et al. (1963) & & 64,72 & Sin indicación \\
\hline WEXELSEN (1928) & & 80 & Estados Unidos \\
\hline
\end{tabular}

Tabla 2. Números cromosómicos en Trifolium medium. 


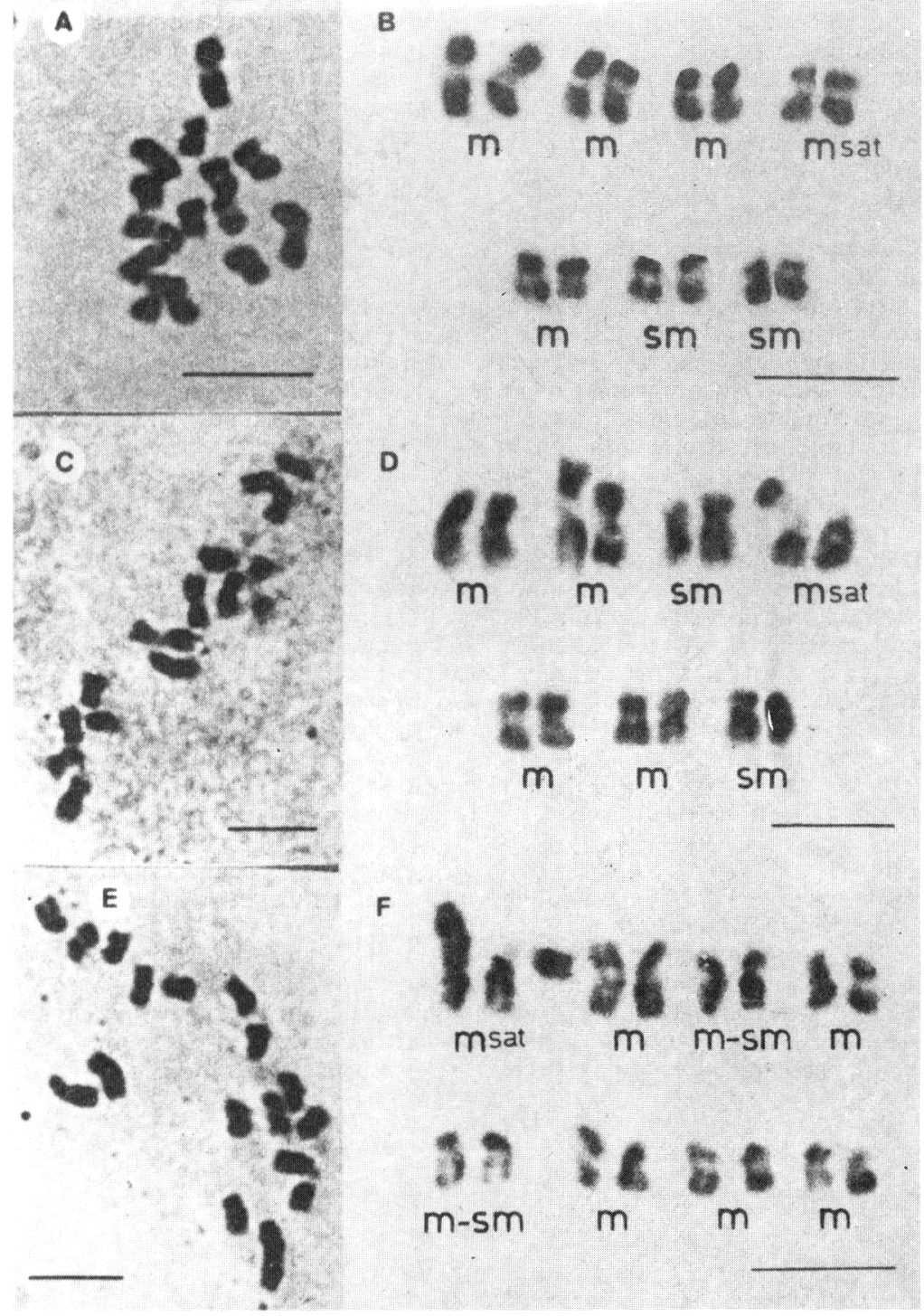

Figura 1. Trifolium pratense var. pratense (GRANADA, UNEX 4790): A) metafase somática 2n=14, B) cariograma. Trifolium pratense subsp. baeticum (CÁDIZ, UNEX 9913): C) metafase somática 2n=14, D) cariograma. Trifolium pallidum (CADIZ, UNEX 4799): E) metafase somática $2 n=16, F$ ) cariograma. Escala $5 \mu \mathrm{m}$. 
El número encontrado coincide con los resultados hallados anteriormente por Taylor et al. (1963 y 1980), Pritchard (1969), Majovsky et al. (1970 a), Zäborsky (1971), Schwer \& Cleveland (1972), Kozuharov et al. (1973 y 1974), Petrova \& Kozuharov (1982 a), y Van Loon \& Van Setten (1982), todos con material extrapeninsular.

En la Península Ibérica el taxon ha sido estudiado cariológicamente por Fernandes y Queiros (1978) y Angulo y Figueras (1983) con material portugués, por lo que el presente recuento es probablemente el primero en realizarse con plantas españolas.

El cariograma elaborado aquí (tab. 11, fig. 2: A y B) es coincidente en el tamaño y ordenación de los cromosomas con el presentado por Zäborsky (1971), y los resultados de Fernandes y Queiros (1978) y Petrova \& Kozuharov (1982 a), diferiendo del que presentan Angulo y Figueras (1983) en cuanto al tamaño relativo de las dos primeras parejas.

Los cromosomas son pequeños o medianamente pequeños, destacando en el cariograma las dos primeras parejas por ser notablemente mayores que las restantes, y es característico en esta especie la presencia de dos pares de cromosomas con constricciones secundarias claras, uno de ellos delimitando netamente el satélite, hecho puesto ya de manifiesto por Angulo y Figueras (1983). Esta particularidad cariológica y otras a nivel morfológico y reproductivo apoyan la segregación de esta especie en una subsección independiente: subsect. Diffusa, propuesta por Muñoz (1992 b).

\section{Subsect. Intermedia (Gibelli \& Belli) Taubert}

\section{Trifolium medium L.}

Material estudiado: GERONA. La Molina, 17. VIII.86, A. Muñoz (UNEX 4841; 2n=84).

Son muy numerosos los autores que han estudiado el taxon, encontrando números cromosómicos muy diversos: $\mathrm{n}=39,48-49$ y $2 \mathrm{n}=48$, $64,72,79,80+0-2 \mathrm{~B}, 82$, c. 84 y c. 126 (tab. 2).

Los números encontrados en esta especie, hasta la fecha, constituyen una serie con número básico $x=8$, en la que al menos se han detectado con exactitud los niveles hexaploide $(2 n=48)$, octoploide $(2 n=64)$, nonaploide $(2 n=72)$, decaploide $(2 n=80)$, dodecaploide $(2 n=96)$ y probablemente hexadecaploide $(2 \mathrm{n}=128)$, niveles a los que se acercan otros números cromosómicos indicados en la bibliografía, y en este estudio (fig. 2: C).

En cuanto al tamaño de sus cromosomas, estos oscilaron entre 1,9 y $3,6 \mu \mathrm{m}$ es decir, pueden considerarse como pequeños o medianamente pequeños, entre los que se cuentan más de un par satelizados (cuatro indicaron Petrova \& Kozuharov, 1982 a).

\section{Subsect. Ochroleuca (Lojac.) Bobrov}

\section{Trifolium ochroleucon Hudson}

Material estudiado: GERONA. Maranges, 11.VIII.86, A. Muñoz (UNEX 9928; $2 n=16$ ).

El recuento efectuado $(2 n=16)$ coincide con los de Bleier (1925 a y b) con material asiático; Majovsky etal. (1970 b) con plantas checoslovacas; Kozuharov et al. (1974) en material búlgaro; ElBaba (1976) con plantas del E del Mediterráneo, y con los números hallados por Fernandes y Santos (1975) y Fernandes et al. (1977) en plantas portuguesas.

Kuzmanov \& Stancev (1972) señalaron, no obstante, el número $2 n=130$, lo cual teniendo en cuenta que tal nivel de ploidía si se encuentra en $T$. pannonicum, especie muy próxima a ésta, hace pensar en la posibilidad de un error en la identificación del material.

Los cromosomas son pequeños o medianamente pequeños y presentan en general baja asimetría, presentando el índice $A_{2}$ uno de los valores más bajos dentro de la sección (tab. 11, fig. 3: A y C). El diagrama representado por Petrova \& Kozuharov (1982 a) discrepa de los resultados obtenidos aquí en el mayor grado de asimetría de sus cromososmas.

Los satélites presentan en la población

\begin{tabular}{lccr}
\hline & $\mathrm{n}$ & $2 \mathrm{n}$ & Origen \\
\hline BLEIER (1925 a) & $\mathrm{c} .48-49$ & & Alemania \\
CODIGNOLA \& MAFFEI (1983) & & 126 & Italia \\
KARPECHENKO (1925) & & $\mathrm{c} .130$ & Rusia \\
KOZUHAROV et al. $(1975)$ & & 126 & Bulgaria \\
KUZMANOV \& STANCEV (1973) & & 128,130 & Bulgaria \\
LÖVE \& LÖVE (1944) & & 126 & Escandinavia \\
MAJOVSKY et al. (1978) & $\mathrm{c} .60-65$ & $128+1-2$ & Checoslovaquia \\
NODA (1946) & & 126 & Japón \\
PETROVA \& KOZUHAROV (1982 a) & & 126 & Bulgaria \\
\hline
\end{tabular}

Tabla 3. Números cromosómicos en Trifolium pannonicum. 


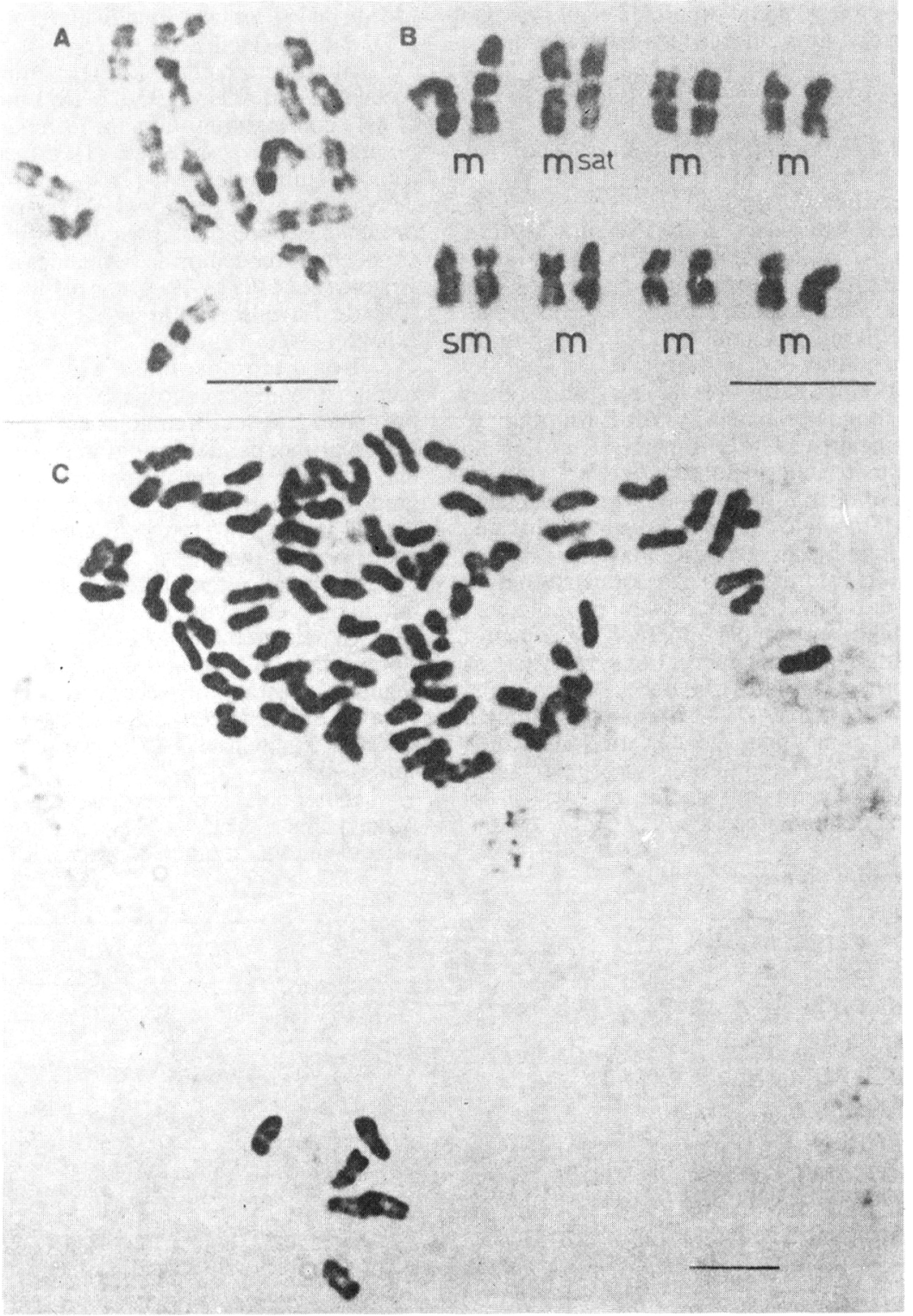

Figura 2. Trifolium diffusum (CÁCERES, UNEX 9906): A) metafase somática 2n=16, B) cariograma. Trifolium medium (GERONA, UNEX 4841): C) metafase somática $2 \mathrm{n}=84$. Escala $5 \mu \mathrm{m}$. 
estudiada un tamaño bastante pequeño, pues sólo suponen el $2,78 \%$ del tamaño total del complemento.

\section{Trifolium pannonicum Jacq.}

Para esta especie no estudiada en el presente trabajo, los distintos autores recogen los números cromosómicos: $n=48-49$ y $2 n=126,128,128 \pm 2$ y c.130 (tab. 3).

\section{Subsect. Macroniotum F. Hermann}

\section{Trifolium rubens $\mathrm{L}$.}

Material estudiado: GERONA. La Molina, 12.VIII.86, A. Muñoz (UNEX 6350; 2n=16).

El resultado obtenido coincide con los recuentos de Karpechenko (1925), Majovsky et al. (1970 a), Nilsson \& Lassen (1971), Holub et al. (1972), Kozuharov et al. (1973), Nielsen (1975) y Petrova \& Kozuharov (1982 a), todos ellos realizados con material del C y E de Europa, por lo que posiblemente sea éste el primer recuento llevado a cabo con material español.

Los cromosomas son todos medianamente pequeños (tab. 11, fig. 3: B y D), siendo los que presentan media más alta de todas las especies estudiadas en este estudio, con asimetría baja y muy uniformes entre parejas, mostrando índices $A_{1}$ y $\mathrm{A}_{2}$ muy bajos en comparación con las restantes especies estudiadas del género. Las características del cariotipo son similares a las del que describieran Petrova \& Kozuharov (1982 a), quienes también señalaron al par más pequeño del cariograma como el único satelizado. Los satélites son relativamente pequeños, abarcando únicamente el $2,75 \%$ del tamaño total del complemento.

Subsect. Lagopodioidea Rouy

\section{Trifolium stellatum $\mathrm{L}$.}

Material estudiado: ALMERÍA. Cabo de Gata, 17.IV.86, J. Herrera y A. Muñoz (UNEX 6399; 2n=14). SEVILLA. Peñón de Algámitas, 5.VI.86, J. A. Mejías y A. Muñoz (UNEX 9910; 2n=14). Morón de la Frontera, Pico Espartero, 25.V.80, M. J. Díez et al. (UNEX 6405; 2n=14).

El número hallado $(2 n=14)$ coincide con los recuentos de Larsen (1956) y De Leonardis et al. (1981) con material.italiano; Pritchard (1969) en plantas israelíes; Nielsen (1975) con material francés; Humphries et al. (1978) con material del N de Marruecos; y Zohary (1971) para material de origen desconocido. En la Península Ibérica se conocen los recuentos de Fernandes et al. (1977) y Angulo et al. (1971 y 1981) con plantas portuguesas, y el de Löve \& Kjellqvist (1974) con material español.

Los cromosomas son pequeños 0 medianamente pequeños (tab. 11, fig. 4: A y B), y presentan grandes diferencias entre parejas, siendo los del primer par del cariograma más de dos veces mayores que los del último, lo cual entraña un grado de asimetría 2B y uno de los valores máximos para el índice $\mathrm{A}_{2}$. Hay tres pares de cromosomas con el brazo largo más de dos veces la longitud que el corto, siendo claramente submetacéntricos, y su índice $\mathrm{A}_{1}$ es el mayor entre las especies estudiadas.

El par satelizado es el cuarto del cariograma y presenta satélites grandes que poseen incluso una segunda constricción secundaria, por lo que ha veces es dificil discernir cual es el centrómero. Los satélites suponen el 7,05 \% del tamaño total del complemento.

Esta población discrepa de la estudiada por Angulo et al. (1971), ya que para estos autores sólo hay dos parejas submetacéntricas, así como por la

\begin{tabular}{|c|c|c|c|}
\hline & $\mathrm{n}$ & $2 n$ & Origen \\
\hline ANGULO et al. (1972 a y b, 1981) & & 14 & España \\
\hline ARUTIONOVA (1940) & & 14 & Rusia \\
\hline BLEIER (1925 a y b) & 8 & & Alemania \\
\hline FERNANDES et al. (1977) & & 14 & Portugal \\
\hline KARPECHENKO (1925) & & 14 & Rusia \\
\hline KOZUHAROV et al. (1972 y 1974) & & 14 & Bulgaria \\
\hline KUZMANOV \& STANCEV (1972) & 14 & & Bulgaria \\
\hline MAJOVSKY et al. (1978) & & 14 & Checoslovaquia \\
\hline NIELSEN (1975) & & 14 & Francia \\
\hline NODA (1946) & 7 & 14 & Japón \\
\hline PETROVA \& KOZUHAROV (1982 a) & 14 & & Bulgaria \\
\hline WEXELSEN (1928) & & 14 & Estados Unidos \\
\hline WIPF (1939) & & 14 & África \\
\hline
\end{tabular}

Tabla 4. Números cromosómicos en Trifolium incarnatum var. incarnatum. 


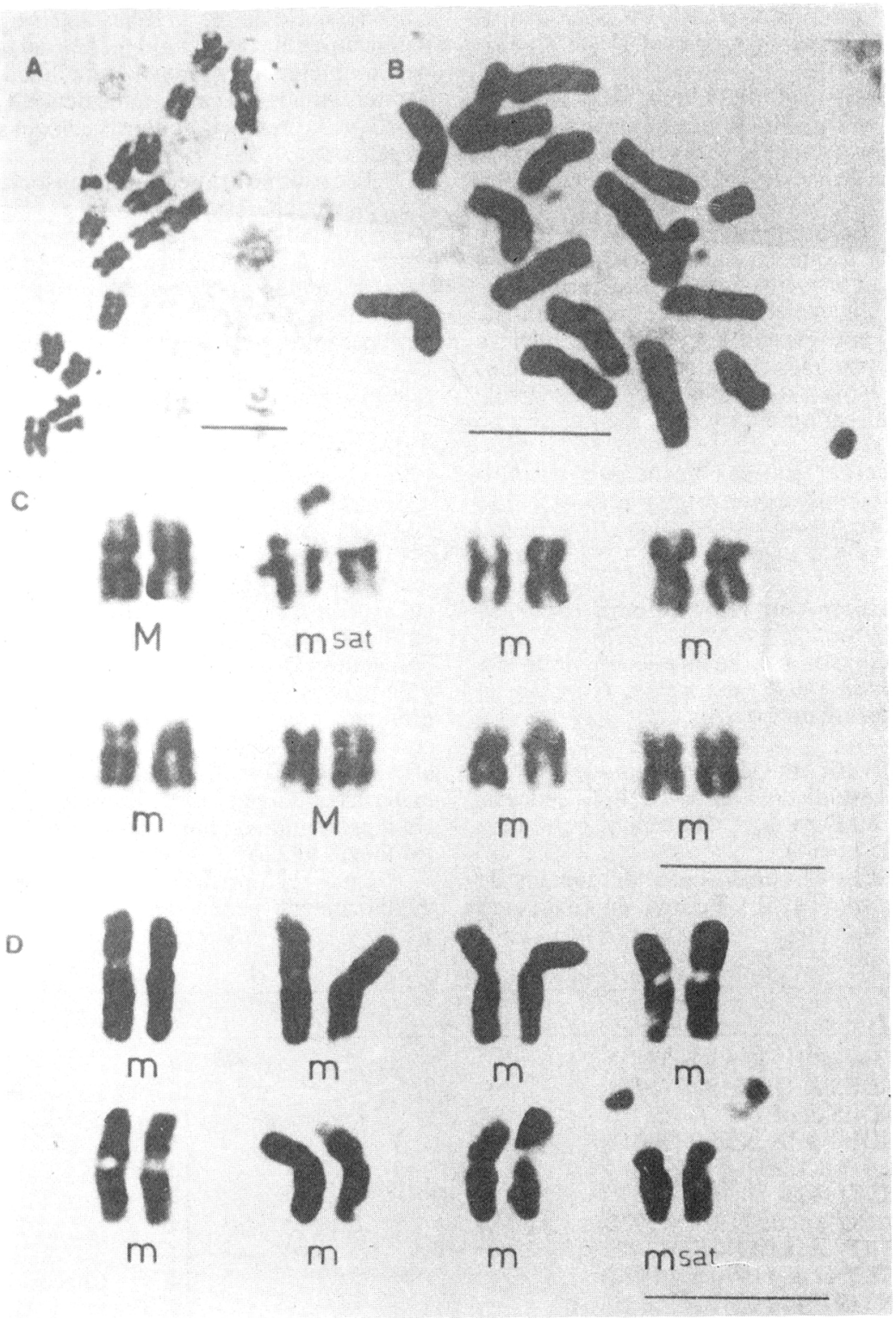

Figura 3. Trifolium ochroleucon (LÉRIDA, UNEX 9928): A) metafase somática 2n=16, C) cariograma. Trifolium rubens (LÉRIDA, UNEX 6350): B) metafase somática $2 \mathrm{n}=16, \mathrm{D})$ cariograma. Escala $5 \mu \mathrm{m}$. 
ordenación de los cromosomas, colocando el par satelizado en segundo lugar, sólo superado en tamaño por un par metacéntrico.

Trifolium incarnatum L. var. incarnatum

Material estudiado: HUESCA. San Juan de Plan, 15.VII.81, P. Montserrat et al. (UNEX 6331; $2 n=14$ ).

El número diploide encontrado $(2 n=14)$ coincide con el encontrado por la mayoría de los autores que han estudiado el taxon (tab. 4), con la sola excepción de Bleier (1925 a y b), que encuentra $\mathrm{n}=8$.

Los cromosomás son pequeños o medianamente pequeños (tab. 11, fig. 4: C y D), siendo las dos primeras parejas las de mayores dimensiones. Su asimetría media es relativamente grande y alcanza el grado $2 \mathrm{~A}, \mathrm{y}$ el índice $\mathrm{A}_{1}$ alcanza uno de los valores más elevados entre las especies estudiadas. El par satelizados ocupa la segunda posición en el cariograma y sus satélites son de tamaño mediano.

Estas características coinciden con las señaladas para el taxon pór Angulo et al. (1972 a) y Petrova \& Kozuharov (1982 a), aunque la asimetría que indican estos es algo mayor.

Trifolium incarnatum var. molinerii (Balb. ex Hornem) Ser.

Para este taxon sólo se ha encontrado la cita de Bartolo et al. (1977) quienes le atribuyen el número cromosómico $2 \mathrm{n}=14$.

Trifolium sylvaticum Gérard ex Loisel.

Material estudiado: SALAMANCA. Ledrada,

27.VI.86, J. A. Devesa, J. Herrera y A. Muñoz (UNEX 9922; $2 \mathrm{n}=14$ ).

El recuento efectuado coincide con los de Kozuharov et al. (1975) y Petrova \& Kozuharov (1982a) con material búlgaro, y Fernandes y Queiros (1978) con plantas portuguesas. Discrepa, por el contrario, de los resultados obtenidos por Angulo et al. $(1970,1981)$ en plantas españolas, que indican $2 n=16$, lo que podría explicarse en base a la consideración de los grandes satélites como una pareja diferente (abarcan el 6,25\% del tamaño total del complemento) o bien a la facilidad con que el par más grande del cariograma tiende a separar sus brazos y a la presencia de constricciones secundarias en estos.

El cariotipo lo integran cromosomas pequeños o medianamente pequeños (tab. 11, fig. 4: E y F), y con asimetría muy variable entre parejas, lo que entraña un grado de asimetría de tipo $2 \mathrm{~B}$ y un alto valor del índice $\mathrm{A}_{2}$. Su índice $\mathrm{A}_{1}$ es también alto, 10 cual relaciona esta especie con las dos anteriores pertenecientes a la subsec. Lagopodioidea.

Subsect. Stenosemium (Celak.) Lojac.

\section{Trifolium striatum L. subsp. striatum}

Material estudiado: ZAMORA. El Cubo de la Tierra del Vino, 27.VI.86, J.A. Devesa et al. (UNEX $1642 ; 2 n=14)$.

El recuento realizado coincide con todos los recuentos anteriormente efectuados para la especie sin especificación subespecífica (tab. 5). Tan sólo en el caso del material portugués estudiado por Fernandes y Queiros (1978), Fernandes et al. (1977), y Fernandes y Santos (1971) se ha comprobado al estudiar los testigos (COI) que el recuento se realizó con material de la subespecie striatum, e igual afirmación, considerando la procedencia del material estudiado, puede hacerse de la población abulense estudiada por Angulo et al. (1972 a y b) (Muñoz, 1992 a).

Los cromosomas son pequeños o medianamente pequeños y presentan en general

\begin{tabular}{lccr}
\hline & $\mathrm{n}$ & $2 \mathrm{n}$ & Origen \\
\hline ANGULO et al. (1972 a y b, 1981) & 7 & 14 & España \\
FERNANDES \& QUEIROS (1978) & & 14 & Portugal \\
FERNANDES et al. (1977) & & 14 & Portugal \\
FERNANDES \& SANTOS (1971) & & 14 & Portugal \\
GADELLA \& KLIPHUIS (1963) & & 14 & Holanda \\
KLIPHUIS (1962) & 14 & Holanda \\
KOZUHAROV et al. (1974 y 1975) & & 14 & Bulgaria \\
KUZMANOV \& STANCEV (1972) & 14 & Bulgaria \\
MAJOVSKY et al. (1970 b y 1974) & & 14 & Francia y Alemaquia \\
NIELSEN (1975) & 14 & Bulgaria \\
PETROVA \& KOZUHAROV (1982 a) & & 14 & Yuggoslavia \\
VAN LOON \& KIEFT (1980) & & 14 & Asia Menor \\
WULF (1939) & & &
\end{tabular}

Tabla 5. Números cromosómicos en Trifolium striatum s.l. 


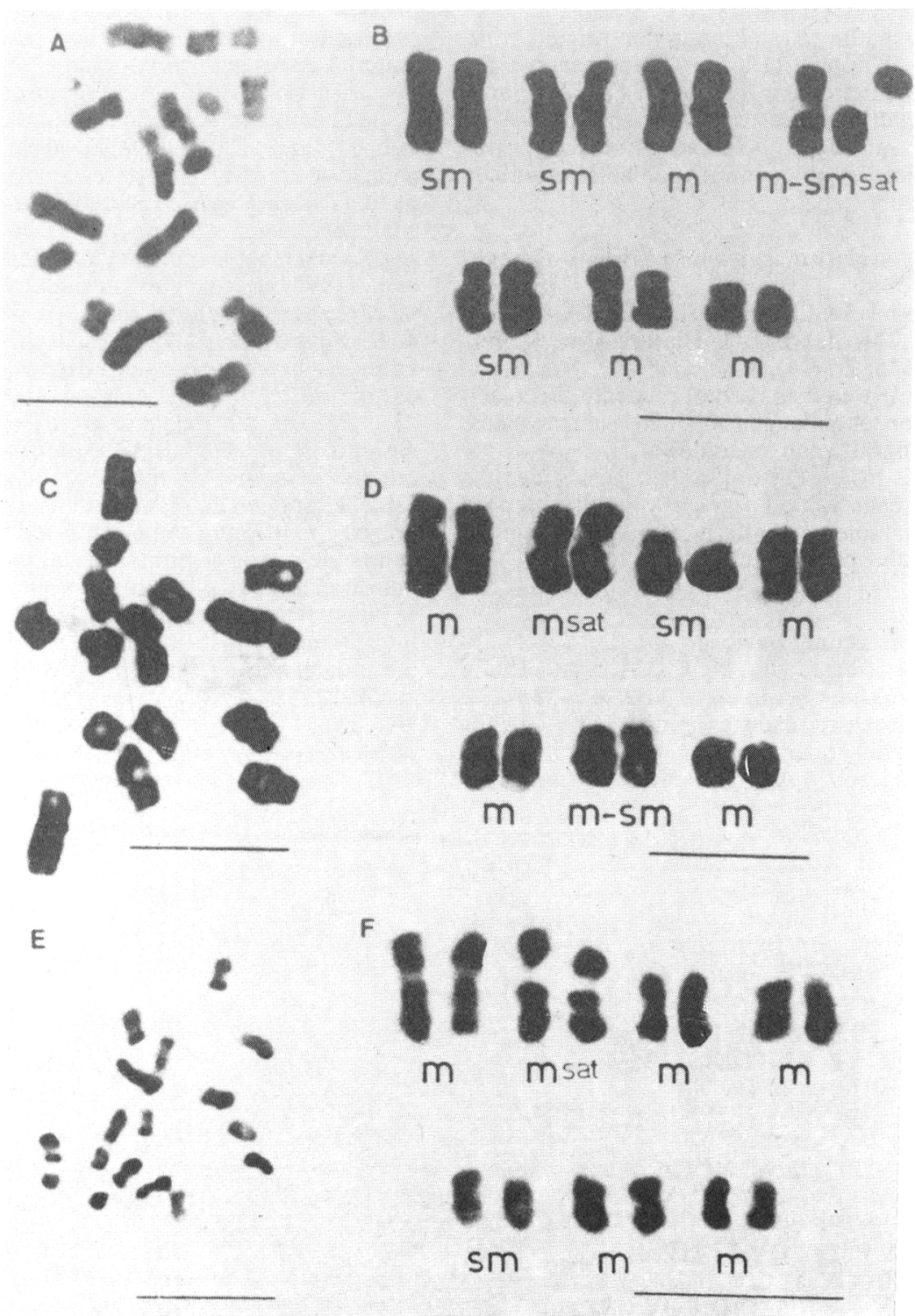

Figura 4. Trifolium stellatum (SEVILLA, UNEX 6405): A) metafase somática $2 \mathrm{n}=14$, B) cariograma. Trifolium incarnatum var. incarnatum (HUESCA, UNEX 6331): C) metafase somática 2n=14, D) cariograma. Trifolium sylvaticum (SALAMANCA, UNEX 9922): E) metafase somática 2n=14, F) cariograma. Escala $5 \mu \mathrm{m}$. 
una baja asimetría (tab. 11, fig. 5: A y D). El par satelizado ocupa la segunda posición en el cariograma, con satélites grandes que suponen el $6,25 \%$ del tamaño total del complemento.

Dichas características coinciden plenamente con las señaladas para el taxon por Angulo et al. (1972 a) y Kliphuis (1962), y discrepan con los hallazgos de Petrova \& Kozuharov (1982 a) por el tamaño relativo de los satélites (los indican de menor tamaño), y la ausencia del par submetacéntrico indicado en la población estudiada en el presente trabajo.

Trifolium striatum subsp. brevidens (Lange) Muñoz

Material estudiado: PONTEVEDRA. Salcido, 25.VI.86, J. A. Devesa, J. Herrera y A. Muñoz (UNEX 1645; $2 \mathrm{n}=14$ ).

También en este caso el recuento efectuado coincide con los hallazgos señalados para la especie en la bibliografía consultada (tab. 5).

Todos los cromosomas pueden considerarse como pequeños (tab. 11, fig. 5: B y E), siendo en los restantes caracteres similares a los que presentan los de la subespecie típica.

\section{Trifolium bocconei Savi}

Material estudiado: HUELVA. Santa Olalla de Cala, 17.VII.86, A. Muñoz (UNEX 9904; 2n=12).

El número hallado es concordante con los indicados por Anderson et al. (1972) en plantas introducidas en U.S.A. y por Petrova \& Kozuharov
(1982 a) con material búlgaro. Discrepa, sin embargo, con el resto de los recuentos señalados en la bibliografía consultada, que indican para esta especie el número $2 \mathrm{n}=14$ (Angulo et al., 1970, con material español; Fernandes y Santos, 1971, en plantas portuguesas, y Böcher et al., 1955, en material de origen desconocido).

El cariotipo lo integran cromosomas pequeños o medianamente pequeños y con baja asimetría (tab. 11, fig. 5: C y F). En él no se han evidenciado parejas satélizadas y sí cierta tendencia en el segundo par del cariograma a la separación de los brazos, el cual posee además constricciones secundarias. Estos resultados coinciden con los obtenidos por Anderson et al. (1972), salvo en las consideraciones sobre el tecer par del cariograma, que ellos al igual Petrova \& Kozuharov (1982 a) consideran satelizado, lo cual en el presente estudio no ha podido corroborarse.

Del estudio del cariograma elaborado por Angulo et al. (1970) se deduce que los dos cromosomas de más que señalan para el taxon $(2 \mathrm{n}=14)$ surgen de la consideración de los dos brazos del tercer par como pareja independiente, en contra incluso de otra de las placas metafásicas que muestran en su propio trabajo en donde fácilmente se cuentan doce cromosomas. Es posible que esta interpretación justifique también los recuentos discordantes de Fernandes y Santos (1971) y Böcher et al. (1955).

Subsect. Camptoneurum F. Hermann

\begin{tabular}{|c|c|c|c|}
\hline & $\mathrm{n}$ & $2 n$ & Origen \\
\hline ANGULO et al. $(1970,1972$ b y 1981$)$ & & 10 & España \\
\hline DAHLGREN et al. (1971) & & 10 & España \\
\hline FERNANDES et al. (1977) & & 10 & Portugal \\
\hline FERNANDES \& SANTOS (1971) & & 10 & Portugal \\
\hline GADELLA \& KLIPHUIS (1963 y 1968 a) & & 10 & Holanda y Francia \\
\hline HUMPHRIESet al. (1978) & 5 & & Marruecos \\
\hline KARPECHENKO (1925) & & 16 & Rusia \\
\hline KLIPHUIS (1962) & & 10 & Holanda \\
\hline KOZUHAROV et al. (1972 y 1975) & & 10 & Bulgaria \\
\hline KUZMANOV \& STANCEV (1972) & & 10 & Bulgaria \\
\hline LARSEN (1960) & & 10 & España \\
\hline LÖVE \& KJELLQVIST (1974) & & 10 & España \\
\hline NIELSEN (1975) & & 10 & Israel e Italia \\
\hline PETROVA \& KOZUHAROV (1982 b) & & 10 & Bulgaria \\
\hline PRITCHARD (1967) & & 10 & Australia \\
\hline RODRIGUES (1953) & & 16 & Portugal \\
\hline VAN LOON (1974 y 1980) & & 10 & España \\
\hline VAN LOON \& KIEFT (1980) & & 10 & Yugoslavia \\
\hline VAN LOON et al. (1971) & & 10 & Francia \\
\hline ZOHARY (1971) & & 16 & Sin indicación \\
\hline
\end{tabular}

Tabla 6. Números cromosómicos en Trifolium scabrum. 


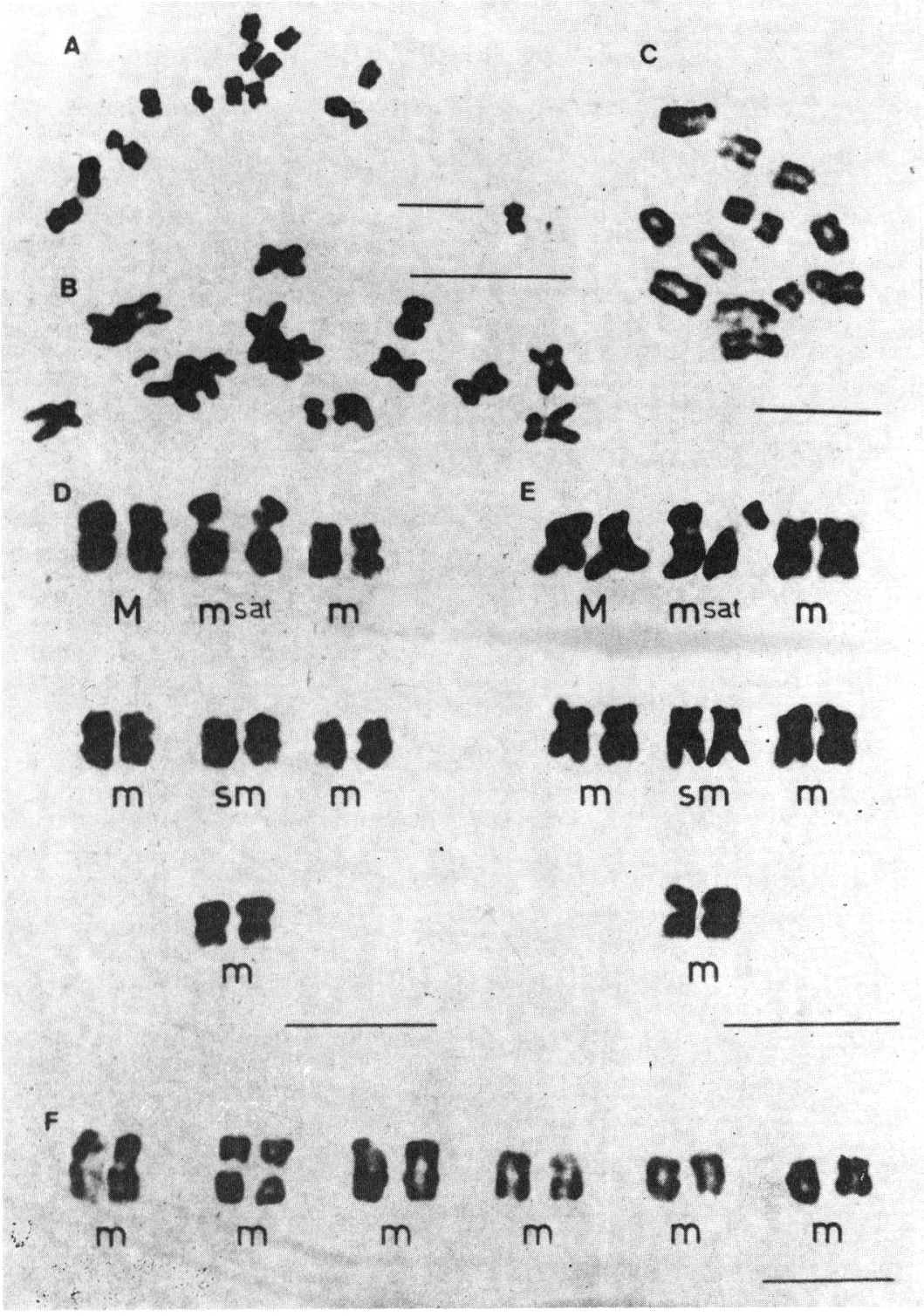

Figura 5. Trifolium stiatum subsp. striatum (ZAMORA, UNEX 1642): A) metafase somática 2n=14, D) cariograma. Trifolium striatum subsp. brevidens (PONTEVEDRA, UNEX 1645): B) metafase somática $2 \mathrm{n}=14$, E) cariograma. Trifolium bocconei (HUELVA, UNEX 9904): C) metafase somática 2n=12, F) cariograma. Escala $5 \mu \mathrm{m}$. 


\section{Trifolium scabrum L.}

Material estudiado: CÓRDOBA. Entre Hornachuelos y San Calixto, 1.VI.81, P. Fernández y I. Porras (SEV 118375;2n=10). SEVILLA. Morón de la Frontera, Pico Esparteros, 21.V.80, M. J. Díez et al. (UNEX 6363; $2 \mathrm{n}=10$ ).

El número encontrado en las dos poblaciones estudiadas $(2 n=10)$ coincide con el hallado por la mayor parte de los autores que han estudiado el taxon (tab. 6) a excepción de Karpechenko (1925), RODRIGUES (1953) y ZOHARY (1971), que señalan $2 n=16$ para esta especie.

Los resultados obtenidos evidencian un cariotipo integrado por cromosomas pequeños y dispares entre parejas (tab. 11, fig. 6: A y C). En las poblaciones estudiadas el par satelizado parece ocupar la primera posición en el cariograma, lo que difiere de las observaciones realizadas por Pritchard (1967) y Angulo et al. (1970), que lo sitúan en segundo lugar. Los satélites son grandes y abarcan el $6,67 \%$ del tamaño total del complemento. El cariotipo mostrado por Angulo et al. (1970) es considerablemente más asimetrico, pues se evidencian en él tres pares de cromosomas submetacéntricos.

Trifolium lucanicum Gasp. ex Guss.

Material estudiado: SEVILLA. Coripe, hacia Zaframagón, 20.V.87, J. A. Mejías y A. Muñoz (UNEX 9927; 2n=10).

El recuento efectuado aquí es probablemente el primero en realizarse para este taxon, el cual ha sido citado por primera vez para la Península recienetemente (MUÑOZ, 1992 b).

Las características del cariotipo (tab. 11, fig. 6: B y D) son muy similares a las del de T. scabrum, taxon muy afín, siendo los cromosomas pequeños o medianamente pequeños y algo mayores que los de la especie anterior. El el par satelizado ocupa también aquí la primera posición del cariograma.

Subsect. Phleoidea (Gibelli \& Belli) Zohary \& Heller
Trifolium gemellum Pourr. ex Willd.

Material estudiado: ESPAÑA. CÁCERES. Plasencia, 27.VI.86, J. A. Devesa, J. Herrera y A. Muñoz (UNEX 9924; 2n=14). ZAMORA. El Cubo de la Tierra del Vino, 27.VI.86, J. A. Devesa, J. Herrera y A. Muñoz (UNEX 1883; 2n=14). PORTUGAL (ALTO ALENTEJO): Entre Sao Vicente y Santa Eulalia, 25.VI.86, J. A. Devesa, J. Herrera y A. Muñoz (UNEX 9903; $2 n=14$ ).

El número encontrado $(2 n=14)$ coincide con los hallados por Angulo et al. (1969 y 1972 b) con material madrileño y por Anderson et al. (1972) en plantas introducidas en U.S.A.

Los cromosomas de esta especie son pequeños o medianamente pequeños y con baja asimetría (tab. 11, fig. 6: E y G), no destacando ningún par sobre el resto del cariotipo ( $A_{1}$ pequeño). El par satelizado ocupa la primera posición del cariotipo y aunque el tamaño de los satélites supone sólo el $3,03 \%$ del tamaño total del complemento, lo cual no lo situa entre los más pequeños del género, su tamaño comparativo con respecto al tamaño total del cromosoma hace que el cariotipo de esta especie sea facilmente identificable.

Todas las características del cariotipo coinciden con las del descrito por Angulo et al. (1969), en el que también se señala el par metacéntrico satelizado.

Trifolium phleoides Pourr. ex Willd.

Para esta especie la bibliografía consultada muestra el número $2 \mathrm{n}=14$ (Anderson et al., 1972; Petrova \& Kozuharov, 1982 b, Angulo et al., 1969 y 1972 b). Las características del cariotipo (Angulo et al., 1969) ponen de manifiesto su gran parecido con T. gemellum en lo concerniente al tamaño relativamente pequeño de los satélites, lo que caracteriza a ambos táxones dentro del género.

Trifolium ligusticum Balb. ex Loisel.

Material estudiado: ESPAÑA. HUELVA. Galaroza, rio Múrtigas, 17.VII.86, A. Muñoz

\begin{tabular}{lccr}
\hline & $\mathrm{n}$ & $2 \mathrm{n}$ & Origen \\
\hline ANDERSON et al. (1972) & & 10 & Estados Unidos \\
ANGULO et al. $(1969$, 1972 b y 1981) & & 10 & España \\
BRITTEN (1963) & 5 & 10 & Sin indicación \\
FERNANDES \& QUEIROS (1978) & & 10 & Portugal \\
KOZUHAROV et al. (1974 y 1975) & & 10 & Bulgaria \\
KUZMANOV \& STANCEV (1972) & 10 & Bulgaria \\
NIELSEN (1975) & 10 & Francia \\
PETROVA \& KOZUHAROV (1982 b) & 10 & Bulgaria \\
PRITCHARD (1967) & & Bulgaria, Estados \\
SCHWER \& CLEVELAND (1972) & & 10 & Sin indicación \\
\hline
\end{tabular}

Tabla 7. Números cromosómicos en Trifolium hirtum 


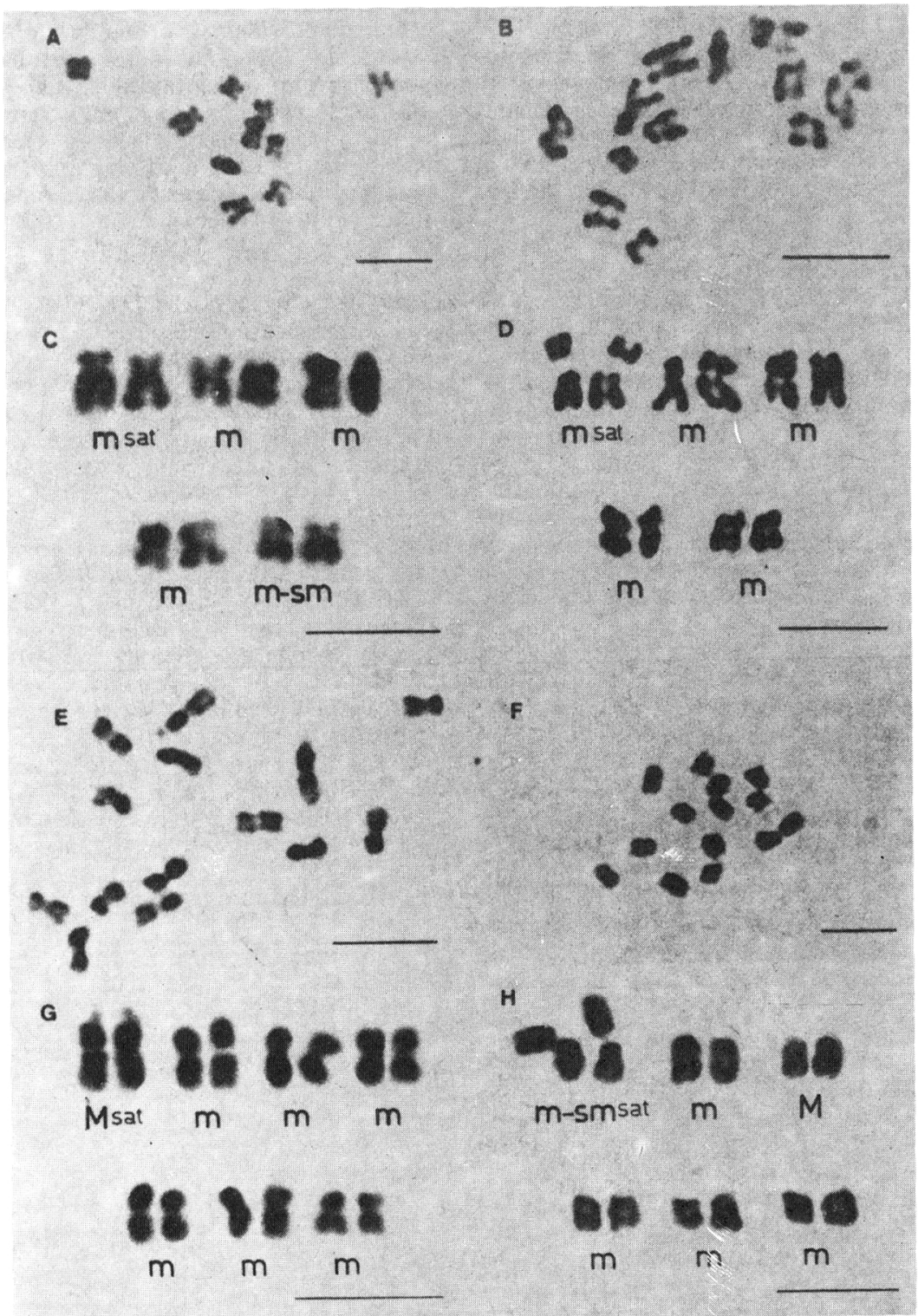

Figura 6. Trifolium scabrum (SEVILLA, UNEX 6363): A) metafase somática 2n=10, C) cariograma. Trifolium lucanicum (SEVILLA, UNEX 9927): B) metafase somática 2n=10, D) cariograma. Trifolium gemellum (ZAMORA, UNEX 1883): E) metafase somática $2 n=14, \mathrm{G}$ ) cariograma. Trifolium ligusticum (LA CORUNA, UNEX 9918): F) metafase somática $2 \mathrm{n}=12, \mathrm{H}$ ) cariograma. Escala $5 \mu \mathrm{m}$. 
(UNEX 9908; 2n=12). PONTEVEDRA. Salcido. 25.VI.86, J. A. Devesa, J. Herrera y A. Muñoz (UNEX 9918; 2n=12). PORTUGAL (BEIRA LITORAL): Coimbra, 24.VI.86, J. A. Devesa, J. Herrera y A. Muñoz (UNEX 4839; $2 n=12$ ).

El número hallado coincide con los recuentos efectuados por Pritchard (1969), Fernandes et al. (1977) y Fernandes y Santos $(1971,1975)$ en plantas portuguesas, y Kozuharov et al. (1975) y Petrova \& Kozuharov (1982 b) con material búlgaro, si bien, discrepa del recuento llevado a cabo por Nielsen $(2 n=14,1975)$ en plantas portuguesas.

El cariotipo lo integran cinco pares de cromosomas pequeños (tab. 11, fig. 6: $\mathrm{F}$ y H), Meta- o metacéntricos y de tamaño similar, y un par medianamente pequeño y satelizado, con satélites muy grandes que abarcan casi el $16,07 \%$ del tamaño total de la dotación: los más grandes de todas las especies estudiadas del género. Los satélites son mayores incluso que el brazo largo del cromosoma y tan grandes o mayores que el resto de los cromosomas, lo que implica una alta asimetría. Este hecho apoya la tesis de Petrova \& Kozuharov (1982 b) de que el recuento $2 n=14$ efectuado por Nielsen (1975) sea consecuencia de la interpretación de los satélites como un par de cromosomas independientes.

El índice $\mathrm{A}_{1}$ es uno de los más bajos observados en las especies estudiadas y por el contrario, el índice $\mathrm{A}_{2}$ es sin duda el mayor.

\section{Subsect. Eikosineurum F. Hermann}

\section{Trifolium hirtum All.}

Material estudiado: ESPAÑA. CÓRDOBA. Entre Hornachuelos y San Calixto, 1.VI.81, P. Fernández y I. Porras (SEV 118410; $2 n=10$ ). PORTUGAL (BEIRA BAIXA): Castelo Branco,
24.VI.86, J. A. Devesa, J. Herrera y A. Muñoz (UNEX 9905; 2n=10).

El recuento efectuado, $2 n=10$, coincide con los resultados obtenidos por los autores que han estudiado previamente el taxon (tab. 7).

Todos los cromosomas son medianamente pequeños y de asimetría similar (tab. 11, fig. 7: A y B), por lo que los índices $\mathrm{A}_{1}$ y $\mathrm{A}_{2}$ son relativamente pequeños. Las parejas son fácilmente identificables, por lo que los resultados obtenidos no muestran grandes diferencias con las observaciones de Angulo et al. (1969), Anderson et al. (1972), Pritchard (1967) y Petrova \& Kozuharov (1982 b).

\section{Trifolium cherleri L.}

Material estudiado: CÁCERES. Plasencia, 27.VI.86, J. A. Devesa, J. Herrera y A. Muñoz (UNEX 9923;2n=10). CÓRDOBA. Fuenteovejuna, El Alcornocado, 6.VI.81, P.Fernández y I.Porras (UNEX 6310; 2n=10). SEVILLA. Peñón de Algámitas, 5.VI.86, J. A. Mejías y A. Muñoz(UNEX 9911; $2 \mathrm{n}=10$ ).

El número diploide encontrado $(2 n=10)$ en todas las poblaciones estudiadas, coincide con los resultados de Pritchard (1967) con material de Israel, Chipre y Argelia; Nielsen (1975) en plantas francesas e israelíes, y Kozuharov et al. (1974 y 1975) y Petrova \& Kozuharov (1982 b) con material búlgaro.

Para la Península Ibérica se conocen los recuentos, también coincidentes, realizados por Fernandes y Santos (1971 y 1975) y Fernandes et al. (1977) con material portugués, Angulo et al. (1969, 1972 b y 1981) en plantas madrileñas y Dahlgren et al. (1971) en plantas de las Islas Baleares.

El cariotipo está formado por cromosomas

\begin{tabular}{|c|c|c|c|}
\hline & $\mathrm{n}$ & $2 n$ & Origen \\
\hline ANGULO et al. (1970) & & 16 & Portugal \\
\hline BLEIER (1925 a y b) & 8 & 16 & Alemania \\
\hline DE LEONARDIS et al. (1981) & & 16 & Italia \\
\hline FERNANDES et al. (1977) & & 16 & Portugal \\
\hline FERNANDES \& SANTOS (1971 y 1975) & & 16 & Portugal \\
\hline HUMPHRIES (1978) & 8 & & Marruecos \\
\hline KARPECHENKO (1925) & & 16 & Rusia \\
\hline KOZUHAROV et al. (1975) & & 16 & Bulgaria \\
\hline NIELSEN (1975) & & 16 & Francia \\
\hline NILSSON \& LASSEN (1971) & & 16 & $\begin{array}{r}\text { Austria y } \\
\text { Yugoslavia }\end{array}$ \\
\hline PETROVA \& KOZUHAROV (1982 b) & & 16 & Bulgaria \\
\hline STRID (1980) & & 16 & Albania \\
\hline VAN LOON \& KIEFT (1980) & & 16 & Yugoslavia \\
\hline ZOHARY (1971) & & 14,16 & Sin indicación \\
\hline
\end{tabular}

Tabla 8. Números cromosómicos en Trifolium lappaceum. 
A
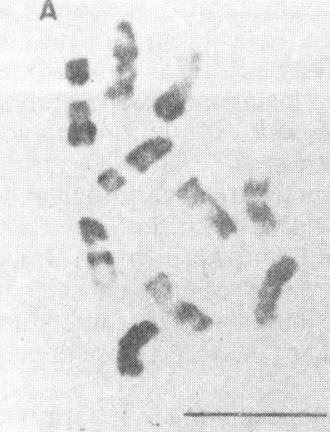

c
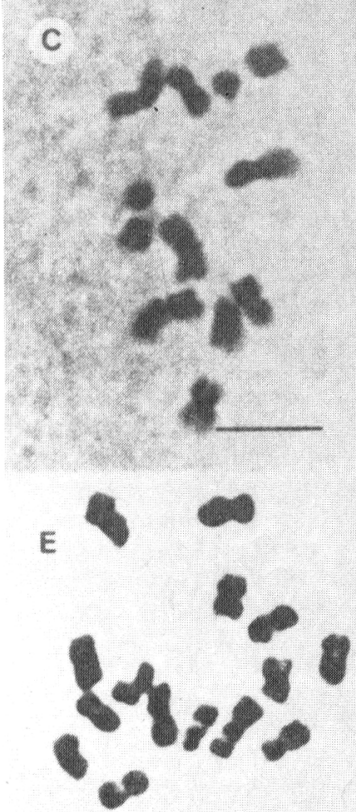

19
B

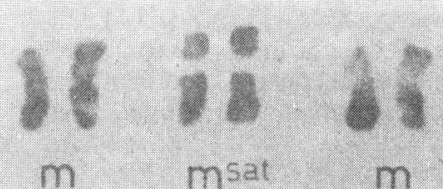

m

D

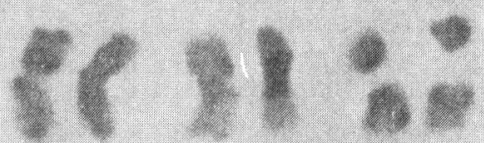

m

m

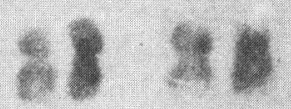

m m

F

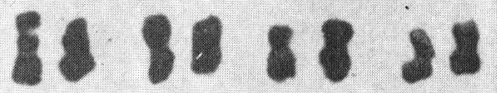

$m$ sat $m$ m $m$

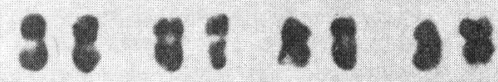

$\mathrm{m}$

m

m

m

Figura 7. Trifolium hirtum (BEIRA BAIXA, UNEX 9905): A) metafase somática 2n=10, B) cariograma. Trifolium cherleri (CÁCERES, UNEX 9923): C) metafase somática $2 n=10, D$ ) cariograma. Trifolium lappaceum (CÁDIZ, UNEX 6338): E) metafase somática $2 n=16$, F) cariograma. Escala $5 \mu \mathrm{m}$. 
medianamente pequeños y de baja asimetría (tab. 11, fig. 7: C y D), siendo muy parecido al de la especie anterior, del que se diferencia no obstante porque el par satelizado ocupa aquí el tercer lugar, y no el segundo como en $T$. hirtum. Todas estas características coinciden con las observables en el idiograma mostrado por Pritchard (1967), y discrepan de las del cariograma mostrado por Angulo et al. (1972 b) en cuanto a la colocación del par satelizado (en primer lugar en su caso) y con respecto al tamaño relativo del par más pequeño, en su caso notablemente menor que el resto de los cromosomas.

\section{Trifolium lappaceum $\mathrm{L}$.}

Material estudiado: CÁDIZ. Zaframagón, 5.VI.86, J. A. Mejías y A. Muñoz (UNEX 6338; $2 n=16$ ). HUELVA. Aljaraque, 3.VII.81, C. López y A. Muñoz (UNEX 6339; 2 n=16). ZAMORA. Cazurra, 27.VI.86, J. A. Devesa, J. Herrera y A. Muñoz (UNEX 6340; $2 n=16$ ).

El número diploide $(2 \mathrm{n}=16)$ encontrado en las tres poblaciones estudiadas coincide con la totalidad de los recuentos llevados a cabo sobre la especie por los distintos autores (tab. 8), resaltando el hecho de los dos números registrados por ZOHARY (1971) para material de origen desconocido.
Los cromosomas son pequeños 0 medianamente pequeños (tab. 11, fig. 7: E y F), no destacando ninguna pareja cromosómica ni por su tamaño ni por su asimetría, a excepción del par satelizado que ocupa el primer lugar del cariograma y. cuyos satélites son relativamente pequeños, que no abarcan sino el 3,95\% del tamaño total de la dotación cromosómica.

El cariograma presentado por Angulo et al. (1970) posee características similares a las del cariotipo observado en las poblaciones estudiadas, si se excluye las asimetrías de al menos a los dos últimos pares, que estos autores caracterizan como submetacéntricos.

Las características cariológicas de esta especie no recuerdan a las de las dos especies anteriores, ni en cuanto al número ni al tamaño de sus cromosomas, aún perteneciendo los tres táxones a la subsect. Eikosineurum, aunque también en este caso sus índices $\mathrm{A}_{1}$ y $\mathrm{A}_{2}$ son pequeños.

Subsect. Lagopodinum (S. F. Gray) Celak.

Trifolium arvense $L$. var. arvense

Material estudiado: ESPAÑA. LEÓN. La Bañeza, 27.VI.86, J. A. Devesa, J. Herrera y A. Muñoz (UNEX 9919; 2n=14). PORTUGAL(TRAS

\begin{tabular}{|c|c|c|c|}
\hline & $\mathrm{n}$ & $2 n$ & Origen \\
\hline AFZAL-RAFII et al. (1985) & \multirow{3}{*}{7} & 14 & Francia \\
\hline ANGULO et al. (1972 a y 1981) & & 14 & Portugal \\
\hline AROHONKA (1982) & & 14 & Finlandia \\
\hline ARUTIONOVA (1940) & \multirow{4}{*}{7} & 14 & Rusia \\
\hline BLEIER (1925 a y b) & & 14 & Alemania \\
\hline BÖCHER \& LARSEN (1958) & & 14 & Austria, Hungría, \\
\hline & & \multicolumn{2}{|c|}{ Dinamarca y Yugoslavia } \\
\hline BÖCHER et al. (1955) & & \multicolumn{2}{|c|}{$\begin{array}{l}14 \text { Austria, Hungría, } \\
\text { Dinamarca y Yugoslavia }\end{array}$} \\
\hline FERNANDES \& SANTOS (1971 y 1975) & & 14 & Portugal \\
\hline GADELLA \& KLIPHUIS (1963) & & 14 & Holanda \\
\hline HOLUB et al. (1972) & & 14 & Checoslovaquia \\
\hline KARPECHENKO (1925) & & 14 & Rusia \\
\hline KLIPHUIS (1962) & & 14 & Holanda \\
\hline KOZUHAROV et al. (1973 y 1975) & & 14 & Bulgaria \\
\hline KUZMANOV \& STANCEV (1972) & & 14 & Bulgaria \\
\hline LÖVE \& LÖVE (1944) & & 14 & Escandinavia \\
\hline MAJOVSKY et al. (1970 a) & & 14 & Checoslovaquia \\
\hline NIELSEN (1975) & & 14 & Francia, Alemania, \\
\hline & \multicolumn{3}{|c|}{ Luxemburgo, Noruega, Suecia y Suiza } \\
\hline PETROVA \& KOZUHAROV (1982 b) & & 14 & Bulgaria \\
\hline POGAN et al. (1983) & & 14 & Polonia \\
\hline STRID (1971) & & 14 & Albania \\
\hline TISCHLER (1934) & & 14 & Alemania \\
\hline UOTILA \& PELLINEN (1985) & & 14 & Finlandia \\
\hline VAN LOON (1974) & & 14 & España \\
\hline
\end{tabular}

Tabla 9. Números cromosómicos en Trifolium arvense. 
A

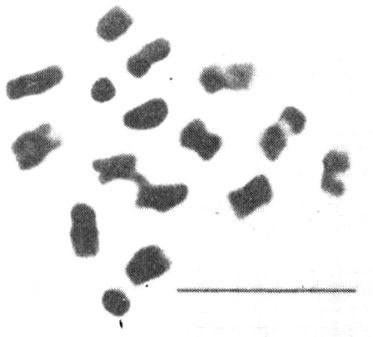

c
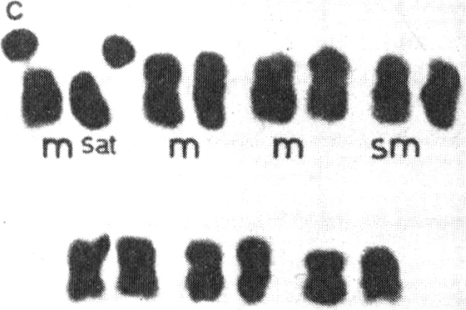

$m$

$m$

$m$

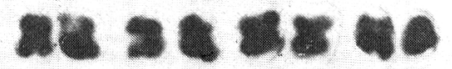

$m$

$m$

$m$ m-sm

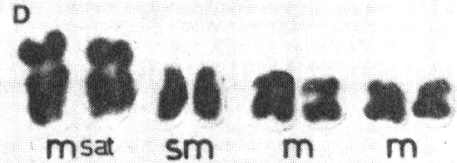

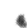

$E$
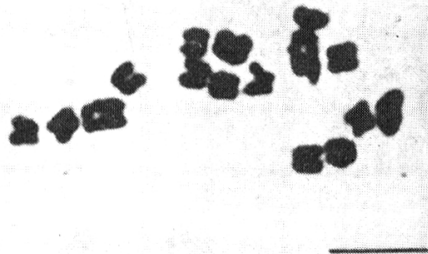

B
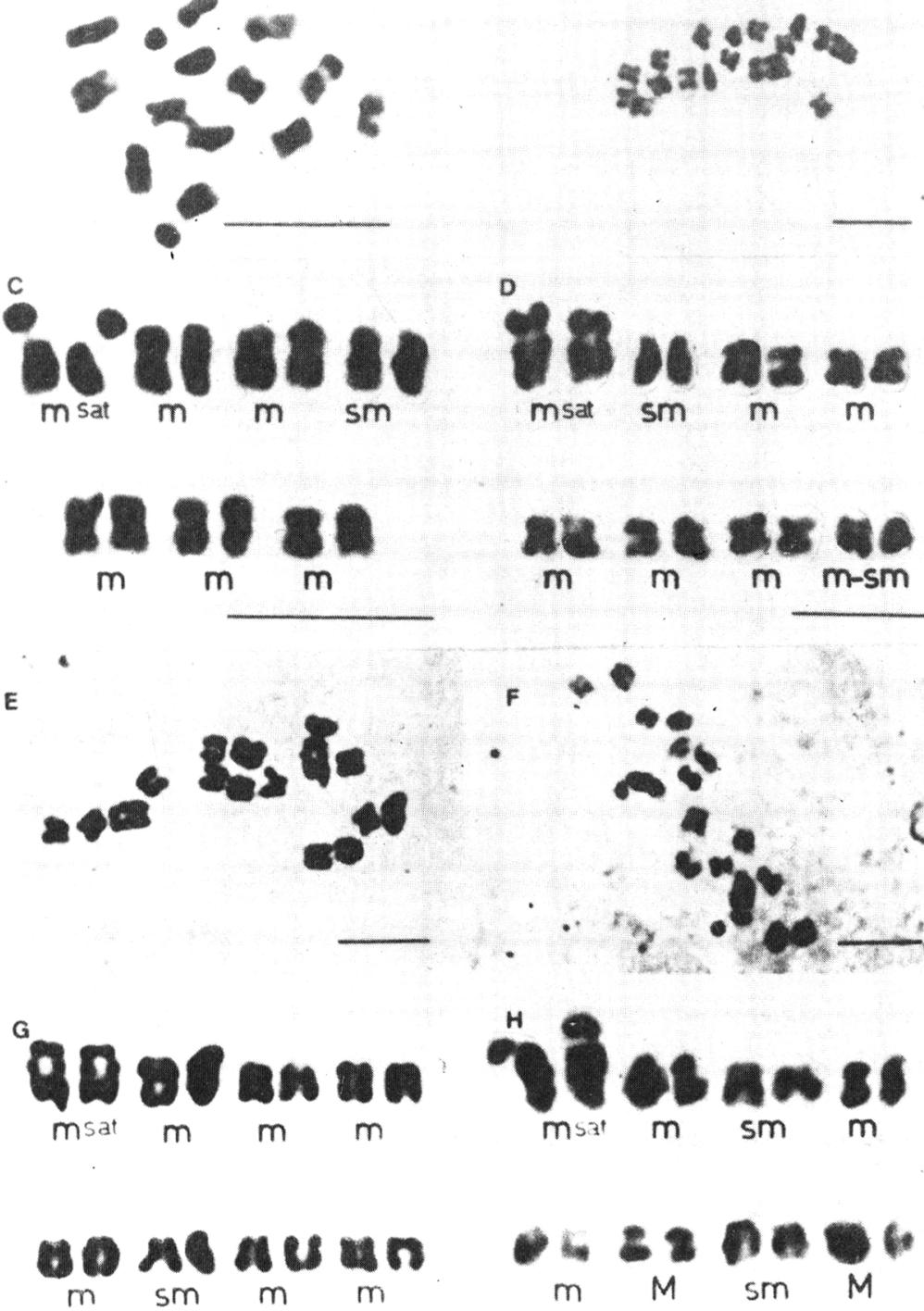

Figura 8. Trifolium arvense var. arvense (TRAS OS MONTES, SEV 118377): A) metafase somática $2 \mathrm{n}=14, \mathrm{C}$ ) cariograma. Trifolium angustifolium (ALMERÍA, UNEX 6278): B) metafase somática 2n=16, D) cariograma. Trifolium alexandrinum (UNEX): E) metafase somática $2 n=16, G$ ) cariograma. Trifolium squamosum (SEVILLA, UNEX 6375): F) metafase somática $2 \mathrm{n}=16, \mathrm{H}$ ) cariograma. Escala $5 \mu \mathrm{m}$. 
OS MONTES). Entre Mirandela y Bragança, 28.VI.82, M. J. Gallego et al. (SEV 118377; $2 \mathrm{n}=14$ ).

El número $2 n=14$ encontrado coincide con el de todos los autores que han estudiado previamente el taxon (tab. 9).

Los cromosomas son pequeños y de tamaño muy uniforme en todo el cariotipo (tab. 11, fig. 8: A y C), destacando por su asimetría tan sólo la cuarta pareja del cariograma. El par satelizado es el de tamaño más grande, suponiendo los satélites el $6,06 \%$ del tamaño total de la dotación cromosóimica. Estos datos coinciden con los resultados obtenidos por Angulo et al. (1972 a).

\section{Subsect. Orthoneurum F. Hermann}

\section{Trifolium angustifolium $L$.}

Material estudiado: ALMERÍA. Cabo de Gata, 17.IV.86, J. Herrera y A. Muñoz (UNEX 6278; $2 n=16$ ). CÁDIZ. Olvera, Puerto Cabañas, 5.VI.86, J. A. Mejías y A. Muñoz (UNEX 6265; 2n=16). HUELVA. Galaroza, río Múrtigas, 17.VII.86, A. Muñoz (UNEX 9926; 2n=16).

El número diploide encontrado $(2 n=16)$ en las dos poblaciones estudiadas coincide con los hallazgos de la mayoría de los autores que han estudiado anteriormente el taxon (tab. 10), y para el que Fernandes y Queiros (1978) detectaron el nivel tetrapoide en material portugués. Sólo Karpechenko (1925) encontró un número diferente: $2 n=14$. Se trata posiblemente del primer recuento en la especie llevado a cabo con plantas de España peninsular, conociéndose la dotación cromosómica en material de las Islas Canarias (2n=16; Larsen, 1960).

Los cromosomas son todos pequeños a excepción de los del primer par del cariograma (tab. 11, fig. 8: B y D), que son medianamente pequeños y están satelizados. La asimetría media es alta, así como los valores de los índices $\mathrm{A}_{1}$ y $\mathrm{A}_{2}$, existiendo en el cariotipo dos parejas de cromosomas submetacéntricos, uno de ellos con una relación entre brazos de 2,8 , por lo que el cariotipo posee un grado de asimetría de tipo 2B (Stebbins, 1971).

Todos los caracteres del cariotipo coinciden con los apuntados por Pritchard (1969) y Petrova \& Kozuharov (1982 b).

\section{Subsect. Alexandrina Zohary}

\section{Trifolium alexandrinum $\mathrm{L}$.}

Material estudiado: Semillas proporcionadas por el Instituto botánico de COIMBRA. (UNEX ;2n=16).

El número diploide encontrado coincide con los hallazgos de Almeida \& Carvalho (1964) y Fernandes et al. (1977), en plantas portuguesas; Wexelsen (1928), en plantas norteafricanas; Sikka et al. (1958), Sareen \& Trehan (1977) y Biv \& Kumari (1979) en material de la India; Putiyevsky \& Katznelson (1970), con plantas portuguesas y australianas, y Noda (1946), Bhaskaran \& Swaminanthan (1958) y Zohary (1971) en plantas de origen no indicado.

El cariotipo lo integran principalmente cromosomas pequeños, a excepción del primer par, que lo constituyen cromosomas medianamente pequeños y satelizados (tab. 11, fig. 8: E y G). Por sus características este cariotipo es similar al descrito para T. angustifolium en lo concerniente al tamaño del par satelizado y al distanciamiento entre constricciones, si bien en este caso este parmucho mayor- no alcanza el doble de la longitud del octavo, por lo que su grado de asimetría es de tipo 2A. Igualmente, se diferencia también en cuanto a la falta de uno de los pares submetacéntricos y al tamaño relativo del segundo par del cariograma, más grande en esta especie que en $T$. angustifolium, donde apenas era mayor que el resto de los cromosomas de las parejas de tamaño pequeño.

\begin{tabular}{lccr}
\hline & $\mathrm{n}$ & $2 \mathrm{n}$ & Origen \\
\hline COLOMBO et al. (1983 a) & & 16 & Italia \\
FERNANDES \& QUEIROS (1978) & 32 & Portugal \\
FERNANDES \& SANTOS (1971) & 16 & Portugal \\
GADELLA \& KLIPHUIS (1972) & 16 & Yugoslavia \\
KARPECHENKO (1925) & 14 & Rusia \\
KOZUHAROV et al. (1974 y 1975) & 16 & Bulgaria \\
LARSEN (1960) & 16 & España \\
NIELSEN (1975) & 16 & Francia e Italia \\
PETROVA \& KOZUHAROV (1982 b) & 16 & Bulgaria \\
PRITCHARD (1969) & 16 & Sin indicación \\
STRID (1971) & 16 & Albania \\
VAN LOON (1974) & 16 & España \\
VAN LOON \& KIEFT (1980) & 16 & Yugoslavia \\
\hline
\end{tabular}

Tabla 10. Números cromosómicos en Trifolium angustifolium. 


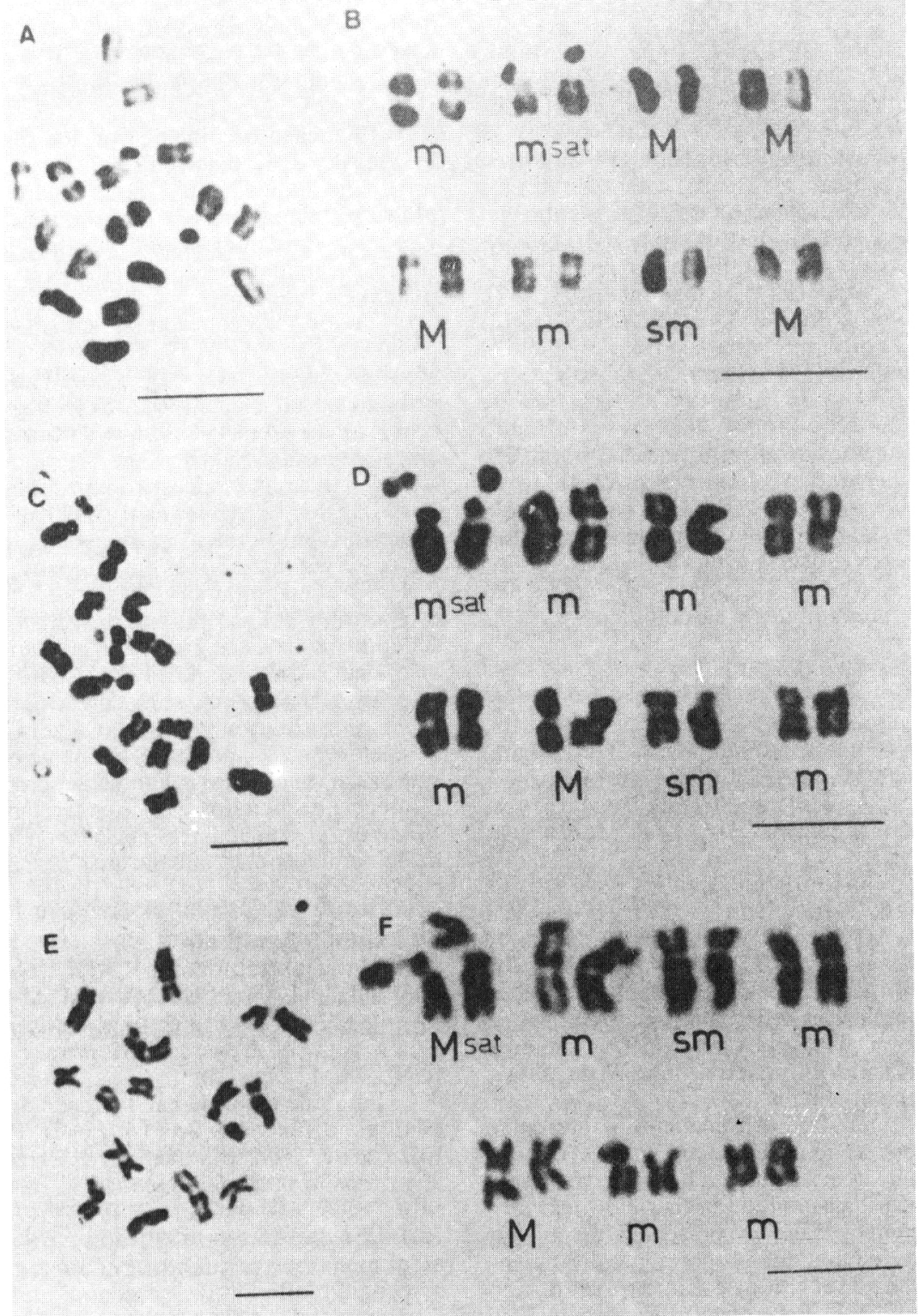

Figura 9. Trifolium squarrosum (SEVILLA, UNEX 6377): A) metafase somática 2n=16, B) cariograma. Trifolium obscurum (CÁDIZ, UNEX 4836): C) metafase somática $2 \mathrm{n}=16$, D) cariograma. Trifolium leucanthum (CÁDIZ, UNEX 9925): E) metafase somática $2 n=14$, F) cariograma. Escala $5 \mu \mathrm{m}$. 


\section{Subsect. Squamosa Zohary}

Trifolium squamosum L.

Material estudiado: CÁDIZ. Los Barrios, Montera del Torero, 9.V.86, A. Muñoz y C. Romero (UNEX 6385; 2n=16). HUELVA. Almonte, El Rocío, Marismas, 3.VII.86, C. López y A. Muñoz (UNEX 6376; 2n=16). SEVILLA. Peñón de Algámitas, 5.VI.86, J. A. Mejías y A. Muñoz (UNEX $6375 ; 2 \mathrm{n}=16$ ).

Los resultados obtenidos en las tres poblaciones estudiadas $(2 n=16)$ coinciden con los obtenidos de Karpechenko (1925) en plantas del SW asiático y con los de Colombo et al. (1979). Para la flora peninsular se conocen los recuentos de Fernandes y Santos (1971), Fernandes et al. (1977), Pritchard (1969) y Putiyevsky \& Katznelson (1972) con material portugués, y para la flora española el de Dahlgren et al. (1971) con material balear.

El cariotipo lo integran siete pares de cromosomas pequeños y un par satelizado claramente mayor que el resto (tab. 11, fig. 8: F y H). Por sus características recuerda al de $T$. angustifolium, del que se diferencia por la ordenación de las parejas de cromosomas pequeños, si bien en ambas especies se han hallado índices $\mathrm{A}_{1}$ y $\mathrm{A}_{2}$ similares (también guardan gran parecido en base a carácteres florales y carpológicos).

\section{Subsect. Urceolata (Lojac.) Zohary}

\section{Trifolium squarrosum $\mathrm{L}$.}

Material estudiado: CÁDIZ. Grazalema, Puerto de los Alamillos, 11.VII.86, A. Muñoz y J. M. Polo (UNEX 6378; $2 n=16$ ). SEVILLA. Coripe, Pico de Zaframagón, 5.VI.86, J. A. Mejías y A. Muñoz (UNEX 6377; $2 n=16$ ).

Para este taxon se conocen los recuentos de Putiyevsky \& Katznelson (1972) en material portugués, y los de Kozuharov et al. (1975) y Petrova \& Kozuharov (1982 b) con material búlgaro, todos ellos coincidentes con los resultados obtenidos aquí. Estos hallazgos difieren de los resultados de Karpechenko (1925) y Labadie (1979), quienes encuentran $2 n=14$ en material del SW de Asia y Argelia, respectivamente.

Los cromosomas son pequeños y presentan pocas diferencias de tamaño entre parejas (tab. 11, fig. 9: A y B), y en cuanto a la asimetría media destacar que es baja debido a la aparición de cuatro parejas metacéntricas. La presencia de un par submetacéntrico con una proporción entre brazos de 2,6 entraña que el grado de asimetría sea de tipo 2A (Stebbins, 1971). Estas observaciones coinciden con los resultados obtenidos por Petrova \& Kozuharov (1982 b).

Al igual que la especie anterior $-T$. squamosum- el cariotipo posee un par submetacéntrico (entre los más pequeños del cariotipo) diferenciándose por otro lado por el tamaño relativo de los primeros tres pares del cariograma, más uniformes en esta especie que en $T$. squamosum, donde el par satelizado es notablemente más grande que los restantes.

\section{Trifolium obscurum Savi}

Material estudiado: CÁDIZ. Peñón de Zaframagón, 5.VI.86, J. A. Mejías y A. Muñoz (UNEX 4836; 2n=16).

El recuento coincide con los resultados de Putiyevsky \& Katznelson (1972) para plantas de origen no indicado; los de Pritchard (1969) en plantas portuguesas y marroquíes, y los de González-Bernáldez et al. $(1973,=$ T. squarrosum subsp. aequidentatum) y Angulo et al. (1981), en plantas portuguesas.

Todos los cromosomas son medianamente pequeños (tab. 11, fig. 9: C y D), destacando tan sólo las dos primeras parejas del cariograma, la primera de ellas satélizada. Sólo hay un par de cromosomas submetacéntricos, con una proporción entre brazos de 2,5.

Estas características difieren de las advertidas . por González-Bernáldez et al. (1973) en cuanto a la simetría cromosómica, ya que estos indican dos parejas con proporciones entre brazos mayores de 2, y un par con proporción 7,5. Los cromosomas de este último serían, por tanto, telocéntricos (el segundo par del cariograma), lo cual no se observa en el material estudiado en el presente trabajo.

Este cariograma se asemeja al de las tres especies anteriores por la existencia de un par submetacéntrico y por la posición que ocupa el par satelizado, uno de los mayores del cariotipo, y se diferencia de aquellos -y más concretamente de $T$. squarrosum, con el que es más afín- por el tamaño de los cromosomas, mucho más grandes en este caso.

\section{Trifolium leucanthum Bieb.}

Material estudiado: CÁDIZ. Grazalema, Puerto de los Alamillos, 11.VII.86, A. Muñoz y J. M. Polo (UNEX 9925; 2n=14).ZAMORA. Cazurra, 27.VI.86, J. A. Devesa, J. Herrera y A. Muñoz (UNEX 6342; 2n=14).

Para este taxon se han indicado dos números básicos diferente: $2 \mathrm{n}=14 \quad(\mathrm{x}=7$; Petrova \& Kozuharov, 1982 b y Napoli \& Zizza, 1984) y $2 \mathrm{n}=16$ ( $\mathrm{x}=8$; Fernandes y Queiros, 1978, Kozuharov et al., 1975, y Angulo et al., 1970 y 1981).

Los cromosomas son pequeños o medianamente pequeños y con tamaños que permiten fácilmente el reconocimiento de las distintas parejas (tab. 11, fig. 9: E y F). En cuanto a la asimetría resaltar la existencia de un par de cromosomas submetacéntricos, con una proporción entre brazos de 2,3, por lo que puede asignarse al cariotipo un grado de asimetría de tipo 2B (Stebbins, 1971).

El primer par de cromosomas posee satélites 
grandes, que suponen el 6,91\% del tamaño total de la dotación cromosómica, a veces no siempre reconocibles por segregarse fácilmente. Tal vez esto explique el conteo de un par de cromosomas de más (Petrova \& Kozuharov, 1982 b). El cariotipo se caracteriza además por presentar un elevado número de constricciones secundarias en varias de las parejas de la dotación (incluso a nivel del satélite).

\section{DISCUSIÓN}

Para facilitar la exposición y la comparación de los resultados obtenidos para cada una de las especies en cuanto al tamaño y asimetría de sus cromosomas y cariotipos, así como de su fórmula, estos se han recogido en la tab. 11.

En el género Trifolium se presentan los números básicos $\mathrm{x}=8,7,6$ y 5 , de ello $\mathrm{x}=8 \mathrm{se}$ considera como el más primitivo, originándose el resto de los números por disploidía (Britten, 1963; Pritchard, 1969; Angulo et al., 1972 b; González-Bernáldez et al., 1973; Petrova \& Kozuharov, 1982 b). Todos los números básicos del género poseen representación en esta sección, donde doce de las especies estudiadas tienen como número básico $\mathrm{x}=8$; nueve $\mathrm{x}=7$; dos $\mathrm{x}=6$ y cuatro $\mathrm{x}=5$.

Todas las especies presentaron una dotación diploide de cromosomas a excepción de las especies perennes $T$. medium y $T$. pannonicum, el primero con $2 \mathrm{n}=84$ (c. $10 \mathrm{x}$, $\mathrm{x}=8$ ) y el segundo - no estudiado- con $n=48-49$ y $2 n=126,128,128 \pm 2$ y c. 130 ( $x=8$, véase tab. $3)$. Para el primero se ha indicado una serie poliploide que con citotipos hexaploides, octoploides, decaploides, dodecaploides y hexadecaploides, y elementos originados por probables desviaciones aneuploides del citotipo decaploide; y para el segundo una serie que incluye elementos dodecaploides y hexadecaploides.

El tamaño cromosómico oscila entre 1,1 y $3,8 \mu \mathrm{m}$, siendo los cromosomas pequeños o medianamente pequeños, y en cuanto al tamaño medio de cada especie oscila entre $1,4 \mu \mathrm{m}$ (T. arvense) y $3,2 \mu \mathrm{m}$ (T. rubens), siendo la media de las especies estudiadas de la sección de $2,14 \mu \mathrm{m}$, la cual fue mucho mayor en las tres especies perennes estudiadas $(2,63 \mu \mathrm{m})$ que para el resto de los táxones anuales $(2,07 \mu \mathrm{m})$.

También en la sección el tamaño total la dotación cromosómica es mayor en las especies perennes (media de $40,53 \mu \mathrm{m}$ ) que en las anuales $(28,08 \mu \mathrm{m})$, siendo $T$. rubens el taxon cuyo cariotipo presentó las mayores dimensiones $(51,0 \mu \mathrm{m})$. Si se relaciona este dato con el número básico de cromosomas, en el caso de las especies anuales, se observa que el tamaño total del cariotipo decrece a media que lo hace el número cromosómico (tab. 12), lo que parece contradecir la idea apuntada por Angulo $e t$ al. (1970, 1971 y 1972 b) de que la reducción en el número cromosómico dentro del género es más consecuencia de reorganizaciones cromosómicas que de pérdida de material génico. El mínimo contenido génico de las especies con $2 n=12$ tal vez explique la escasez de especies con este número (Zohary \& Heller, 1984).

La asimetría media de las especies estudiadas oscila entre 1,2 (T. striatum subsp. striatum, T. gemellum, $T$. ligusticum y $T$. cherleri) y 1,8 (T. stellatum), siendo la media global 1,36, no habiéndose observados diferencias entre las especies anuales $(1,36)$ y las perennes $(1,35)$. La mayor parte de los cromosomas son metacéntricos, si bien en las subsecciones Lagopodioidea, Lagopodium, Orthoneurum, Alexandrina, Squamosa y Urceolata es característica la existencia de al menos un par submetacéntrico, con una proporción entre brazos superior a 2, por lo que en todas ellas las especies muestran un grado de asimetría de tipo 2 (Stebbins, 1971).

Seis especies presentan un grado de asimetría de tipo B (Stebbins, 1971), es decir, el primer par del cariograma es más de dos veces la longitud del último, pudiéndose destacar en este sentido la estrecha relación existente entre $T$. angustifolium y $T$. squamosum, cuyos cariotipos lo integran un par de cromosomas satelizados relativamente grandes y siete pares pequeños de tamaño muy similar entre sí.

En la fig. 10 se observa la concordancia

\begin{tabular}{ccc}
\hline $2 \mathrm{n}$ & $\mathrm{n}^{\mathrm{o}}$ de especies & Tamaño medio $(\mu \mathrm{m})$ \\
\hline 10 & 4 & 25,7 \\
12 & 2 & 23,4 \\
14 & 7 & 27,8 \\
16 & 8 & 30,7 \\
\hline
\end{tabular}

Tabla 12. Tamaños medios del complemento total de las distintas especies anuales agrupadas por números básicos. 


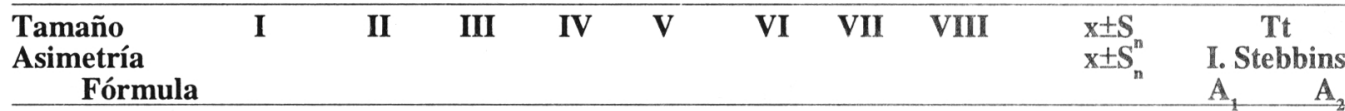

Trifolium pratense susbsp. pratense var. pratense

$\begin{array}{llllll}\text { Tamaño } & 3,1 & 2,9 & 2,6 & 2,4(0,8) & 2,3\end{array}$

$\begin{array}{llllll}\text { Asimetría } & 1,2 & 1,3 & 1,1 & 1,1 & 1,9\end{array}$ $8 m+2 m s a t+4 s m$

Trifolium pratense subsp. baeticum

$\begin{array}{llll}\text { Tamaño } & 3,2 & 3,0 & 2,8\end{array}$

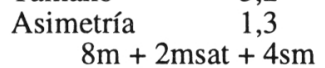

Trifolium pallidum

Tamaño 3,0 $(1,2)$

$\begin{array}{lccc}\text { Asimetría } & 3,0(1,2) & 2,5 & 2,2 \\ & 1,3 & 1,4 & 1,7\end{array}$ $10 m+2 m s a t+4 m-s m$

Trifolium diffusum

$\begin{array}{ll}\text { Tamaño } & 2,9 \\ \text { Asimetría } & 1,6\end{array}$ $12 m+2 m s a t+2 s m$

Trifolium ochroleucon

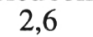

$\begin{array}{ll}2,2 & 2,2 \\ 1,2 & 1,8\end{array}$

$2,5 \pm 0,33 \quad 35,4$

$1,4 \pm 0,31 \quad 1 \mathrm{~A}$

$0,286 \quad 0,132$

$2,5 \pm 0,43 \quad 35,4$

$1,5 \pm 0,28 \quad 1 \mathrm{~A}$

$0,333 \quad 0,172$

$4 M+10 m+2 m s a t$

Trifolium rubens

Asimetría

$3,6 \quad 3,4 \quad 3,4$

$14 m+2 m s a t$

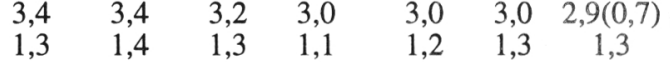

at

Trifolium stellatum

2,7

$, 27 \quad 2,6 \quad 2,5(1,1) \quad 2,1$

$1,9 \quad 1,1$

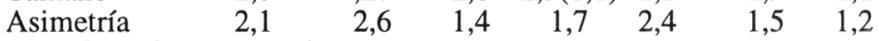
$6 m+2 m-s m s a t+6 s m$

Trifolium incarnatum

$\begin{array}{llllllll}\text { Tamaño } & 3,2 & 2,7(0,8) & 2,3 & 2,2 & 2,2 & 2,2 & 1,8\end{array}$

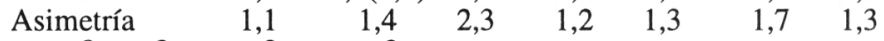
$8 m+2 m s a t+2 m-s m+2 s m$

Trifolium sylvaticum

$\begin{array}{llllllll}\text { Tamaño } & 2,5 & 2,0(0,7) & 1,6 & 1,4 & 1,3 & 1,2 & 1,2\end{array}$

$\begin{array}{llllllll}\text { Asimetría } & 1,5 & 1,3 & 1,2 & 1,3 & 2,0 & 1,2 & 1,6\end{array}$ $10 m+2 m s a t+2 s m$

Trifolium striatum subsp. striatum

$\begin{array}{llll}\text { Tamaño } & 2,3 & 2,2(0,8) & 1,9\end{array}$

$\begin{array}{ccc}\text { Asimetría } & 1,0 \quad 1,1 \quad 1,1 \\ 2 \mathrm{M}+8 \mathrm{~m}+2 \mathrm{msat}+2 \mathrm{sm} & \end{array}$ $2 \mathrm{M}+8 \mathrm{~m}+2 \mathrm{msat}+2 \mathrm{sm}$
olium striatum subsp. brevidens

Trifolium striatum subsp. brevidens

$\begin{array}{lccccccc}\text { Tamaño } & 1,8 & 1,8(0,5) & 1,6 & 1,6 & 1,6 & 1,5 & 1,3 \\ \text { Asimetría } & 1,0 & 1,2 & 1,1 & 1,3 & 1,9 & 1,3 & 1,1\end{array}$ $2 \mathrm{M}+8 \mathrm{~m}+2 \mathrm{msat}+2 \mathrm{sm}$

Trifolium bocconei

$\begin{array}{lllllll}\text { Tamaño } & 2,6 & 2,3 & 2,3 & 1,9 & 1,6 & 1,5 \\ \text { Asimetría } & 1,2 & 1,2 & 1,5 & 1,1 & 1,1 & 1,4\end{array}$ $12 \mathrm{~m}$

Trifolium scabrum

$\begin{array}{llllll}\text { Tamaño } & 2,2(0,6) & 1,9 & 1,9 & 1,6 & 1,4\end{array}$

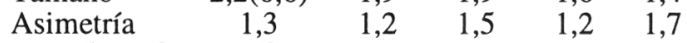
$6 m+2 m s a t+2 m-s m$

Trifolium lucanicum

$\begin{array}{llllll}\text { Tamaño } & 2,7(1,0) & 2,7 & 2,5 & 1,8 & 1,7\end{array}$

$\begin{array}{lccccc}\text { Asimetría } & 1,4 & 1,5 & 1,1 & 1,2 & 1,5\end{array}$
$1,8 \quad 1,6 \quad 1,5 \quad 1,5$

$1,1 \quad 1,3$

(1)
$2,3 \pm 0,38 \quad 36,4$

$1,3 \pm 0,25 \quad 1 \mathrm{~A}$

$0,231 \quad 0,200$

$2,2 \pm 0,21 \quad 35,2$

$1,3 \pm 0,21 \quad 1 \mathrm{~A}$ $0,231 \quad 0,095$

$3,2 \pm 0,24 \quad 51,0$

$1,3 \pm 0,11 \quad 1 \mathrm{~A}$ $0,231 \quad 0,075$

$2,2 \pm 0,54 \quad 31,2$

$1,8 \pm 0,49{ }^{2 B} 0,444 \quad 0,245$

$2,4 \pm 0,42 \quad 33,2$

$1,5 \pm 0,38 \quad 2 \mathrm{~A}$

$0,333 \quad 0,175$

$1,6 \pm 0,45 \quad 22,4$

$1,4 \pm 0,27 \quad 2 \mathrm{~B}$

$0,286 \quad 0,281$

$1,8 \pm 0,30 \quad 25,6$

$1,2 \pm 0,25 \quad 1 \mathrm{~A}$

$0,167 \quad 0,167$

$1,6 \pm 0,16 \quad 22,4$

$1,3 \pm 0,28 \quad 1 \mathrm{~A}$

$0,231 \quad 0,100$

$2,0 \pm 0,40 \quad 24,4$

$1,3 \pm 0,15 \quad 1 \mathrm{~A}$

$0,231 \quad 0,200$

$1,8 \pm 0,28 \quad 18,0$

$1,4 \pm 0,19 \quad 1 \mathrm{~A}$ $0,286 \quad 0,156$

$2,3 \pm 0,44 \quad 22,8$

$1,3 \pm 0,16 \quad 1 \mathrm{~A}$

$0,231 \quad 0,191$ $\begin{array}{lllc}1,6 & 1,6 & 2,1 \pm 0,46 & 32,8 \\ 1 & 1,3 & 1,4 \pm 0,22 & 1 \mathrm{~A} \\ 0,286 & 0,219\end{array}$

$8 m+2 m s a t$ 


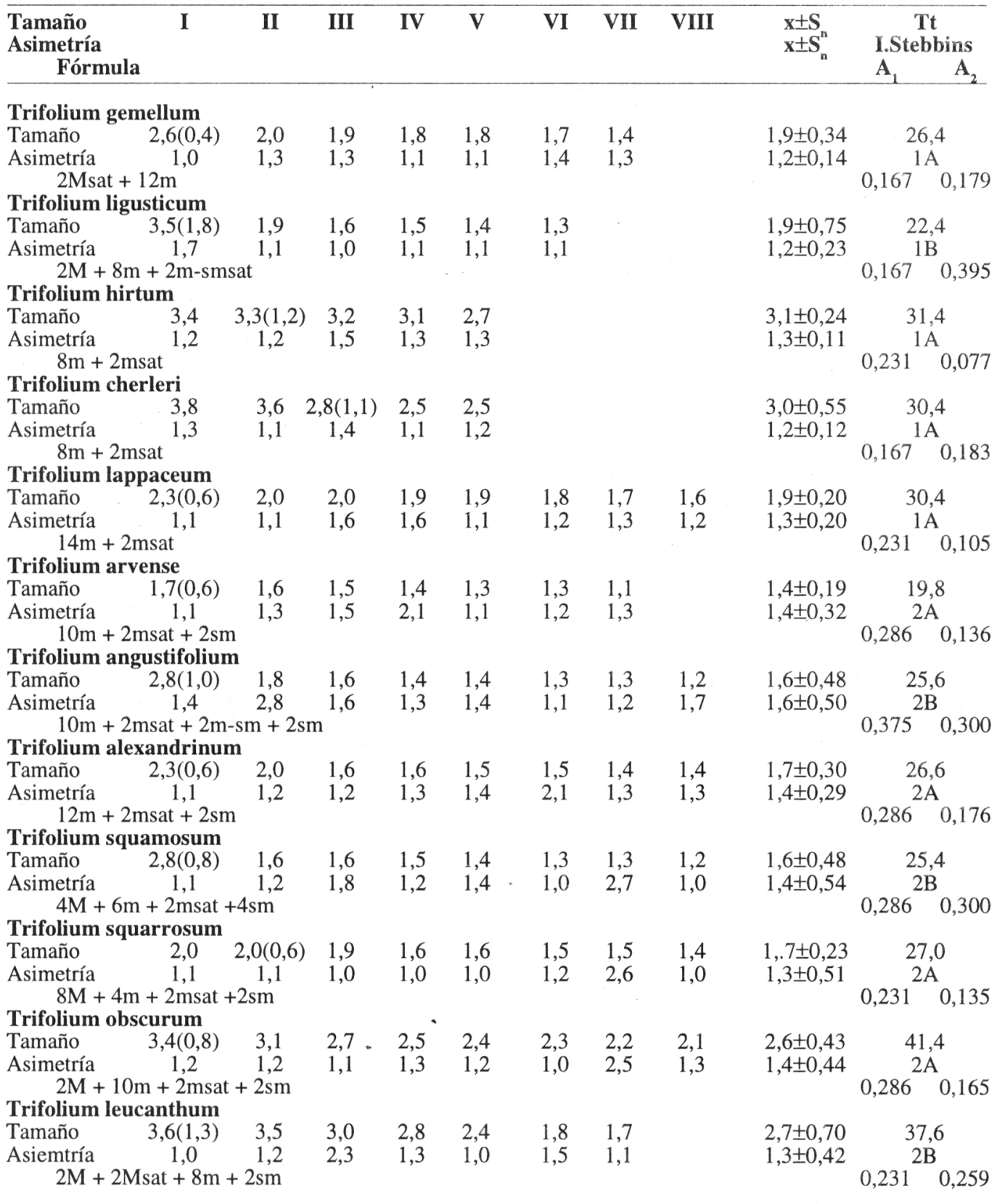

Tabla 11. Datos cariológicos de las especies estudiadas, referidos a sus tamaños cromosómicos (en micras, entre paréntesis figura el tamaño del satélite) y asimetría. ( \pm Sn: media \pm desviación típica; Tt: tamaño total del cariotipo; I. Stebbins: índice de asimetría de Stebbins (1971); A y A : índices de asimetría de Romero (1986). 


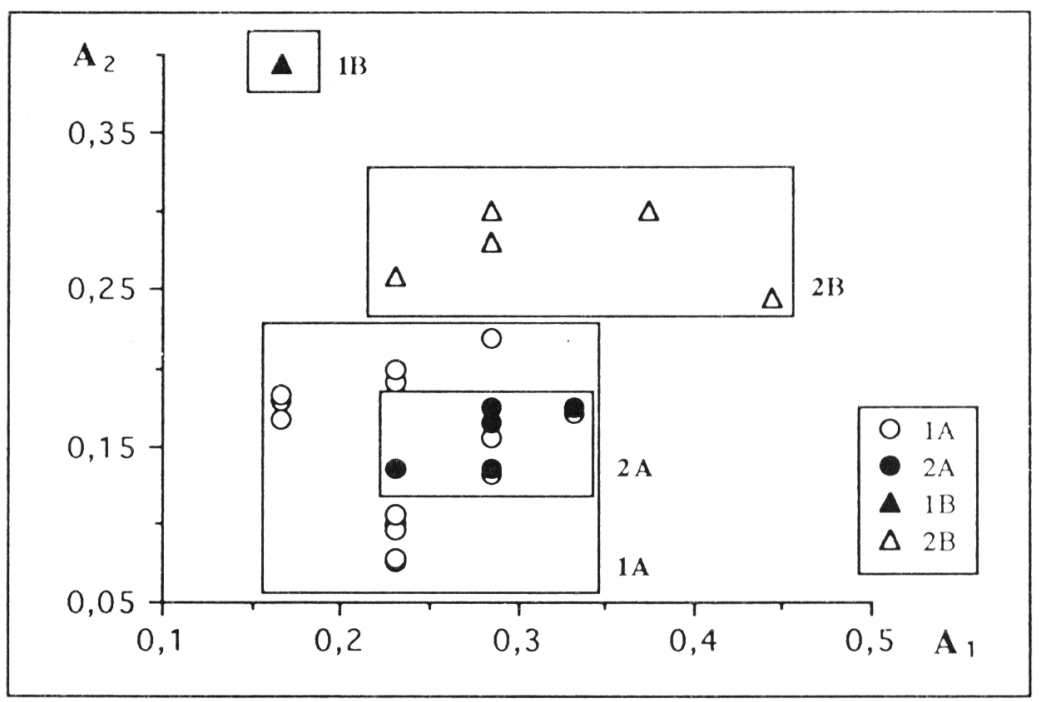

Figura 10. Representanción gráfica de los valores obtenidos en las distitas especies para los índices $\mathrm{A}_{1} \mathrm{y}$ $\mathrm{A}_{2}$ (Romero, 1986), y la agrupación de éstas dependiendo de su grado de asimetría (Stebbins, 1971).

observada entre los grados de asimetría descritos por Stebbins (1971) y los índices dados por Romero (1986). Se puede apreciar, la enorme utilidad de estos índices, en cuanto a la separación de los distintos grados, y mayormente en su aplicación entre las especies con un mismo grado de asimetría.

Todas las especies estudiadas poseen un sólo par de cromosomas satelizados (no confirmado en T. bocconei), cuya ubicación en el cariograma resultó ser diferente: en el primer par en doce; en el segundo en siete; en el tercero en una; en el cuarto en dos, y sólo en $T$. rubens ocupa el último lugar. El tamaño de los satélites oscila entre $0,4 \mu \mathrm{m}$ (v.g. en $T$. gemellum) y $1,8 \mu \mathrm{m}$ en $T$. ligusticum, especie esta última en la que suponen el 16,07\% del tamaño total de la dotación cromosómica, mayor incluso que algunos de los pares cromosómicos. En $T$. gemellum y $T$. phleoides el pequeño tamaño de los satélites respecto del tamaño de los cromosomas que los portan, constituye un fenómeno que no sólo pone de manifiesto la afinidad entre ambos sino que permite además, su caracterización citológica (Angulo et al., 1969). Los satélites suponen por término medio el $6,07 \%$ del tamaño total del cariotipo, siendo máximo en las especies anuales $(6,53 \%)$ y mínimo en las perennes $(2,97 \%)$.
AGRADECIMIENTOS. A los Doctores T. Luque y J. A. Mejías (Universidad de Sevilla) por sus enseñanzas y consejos en el inicio de este trabajo, así como a los Profesores Doctores J. A. Devesa (Universidad de Extremadura) y S. Talavera (Universidad de Sevilla) por la dirección del mismo.

\section{BIBLIOGRAFÍA}

AFZAL-RAFIL, Z., J. VIANOT, M. RAMADE \& M.P. BOURREIT -1985- Analyses des charactères caryologiques et écologiques des quelques taxons dans les massifs du Labéron, de Lure et de Mont-Ventoux. Rev. Cytol. Biol. Vég. Bot., 8:33-62.

ALMEIDA, J.L.F. \& H.P. CARVALHO -1964Trevos autotetraploides III. O caso de Trifolium alexandrinum L.. Agron. Lusit., 24:45-76.

ANDERSON, M.K., N.L. TAYLOR \& G.B. COLLINS -1972- Somatic chromosome numbers in certain Trifolium species. Canad. Jour. Genet. Cytol., 14:139-145.

ANGULO, M.D. y M.C. FIGUERAS -1983- Nota complementaria sobre números cromosómicos y de sacos polínicos en especies de Trifolium. Genét. Ibér., 35:75-86.

ANGULO, M.D., M.C. FIGUERAS y A.M. SÁNCHEZ DE RIVERA -1981- Estudios cariohistológicos en el género Trifolium. Bol. Soc. Brot. sér. 2, 53:875-883. 
ANGULO, M.D., A.M. SÁNCHEZ DE RIVERA y F. GONZÁLEZ-BERNÁLDEZ -1969- Estudios cromosómicos en el género Trifolium. Anal. Est. Exp. Aula Dei, 9:97-110.

ANGULO, M.D., A.M. SÁNCHEZ DE RIVERA y F. GONZÁLEZ-BERNÁLDEZ -1970- Estudios cromosómicos en el género Trifolium III. Bol. Soc. Brot. sér. 2, 44:13-26.

ANGULO, M.D., A.M. SÁNCHEZ DE RIVERA y F. GONZÁLEZ-BERNÁLDEZ -1971- Estudios cromosómicos en el género Trifolium $\mathrm{V}$. Bol. Soc. Brot. sér. 2, 45:253-267.

ANGULO, M.D., A.M. SÁNCHEZ DE RIVERA y F. GONZÁLEZ-BERNÁLDEZ - 1972 a- Estudios cromosómicos en el género Trifolium VI. Lagascalia, 2(1):3-13.

ANGULO, M.D., A.M. SÁNCHEZ DE RIVERA y F. GONZÁLEZ-BERNÁLDEZ - 1972 b- Estudios cromosómicos en el género Trifolium VII. Revisión cariológica sobre especies de la subsección Probatostoma. Genét. Ibér., 24:305324.

AROHONKA, T. -1982- Chromosome count of vascular plants of the island Seili in Navo, SW Finland. Turum Yliop. Biol. Laitok. Julkais., $3: 1-12$.

ARUTIONOVA, A. -1940- Chromosome morphology in certain species of clover. Compt. Rend. Acad. URSS., 27:825-827.

BARTOLO, G., S. BRULLO, G. MAJORANA \& P. PAVONE - 1977- Numeri cromosomici per la Flora Italiana: 315-328. Inf. Bot. Ital., 9:7187.

BHASKARAN, S. \& M.S. SWAMINATHAN 1958- Polyploidy and the genesis of the leguminous root nodule. Nucleus, 1:75-88.

BIV, S.S. \& S. KUMARI -1979- Cytological evolution of the leguminous flora of the Punjal plain. Rec. Res. Pl. Sci. (New Delhi), 7:252-260

BLEIER, H. -1925 a- Chromosomenstudien bei der Gattung Trifolium. Jahrb. Wiss. Bot., 64(4):604-636.

BLEIER, H. -1925 b- Chromosomenzahlen und Kern volumina in der Gattung Trifolium. Ber. Deutsch Bot. Ges., 43(5):236-238.

BÖCHER, T.W. \& K. LARSEN -1958- Experimental and cytological studies on plant species IV. Further studies in short-lived herbs. Biol. Meddel. Kong. Danske Vid. Selsk, 10:1-24.

BÖCHER, T.W., K. LARSEN \& K. RAHN - 1955 Experimental and cytological studies on plant species II. Trifolium arvense and some other pauciennial herbs. Biol. Meddel. Kong. Danske Vid. Selsk, 8(3):1-31.

BRAGDO, H. -1955- Production of polyploids by colchicine. Euphytica, 4:76-82.

BREWBAKER, J.L. -1952- Colchicine induction of tetraploids in Trifolium species. Agron. Jour., 44:592-594.

BRITTEN, E.J. -1963- Chromosome numbers in the Genus Trifolium. Cytologia, 28(4):428449.

BUTTERFASS, T. -1960- Ploidie und Chloroplastenzahlen. Ber. Deutsch. Bot. Ges., 72(10):440-451.

CINCURA, F. -1965- Cytotaxonomické vyhodnotenie Trifolium sarosiense Hazsl. Biológia (Bratislava), 20(4):300-305.

CODIGNOLA, A. \& M. MAFFEI -1981- Numeri cromosomici per la Flora Italiana: $846-849$. Inf. Bot. Ital., 13:179-181.

CODIGNOLA, A. \& M. MAFFEI -1983- Numeri cromosomici per la Flora Italiana: 837-940. Inf. Bot. Ital., 15:35-38.

COLOMBO, P., C. MARCEÑO \& R. PRISCIOTTA - 1979- Numeri cromosomici per la Flora Italiana: 662-675. Inf. Bot. Ital., 11:315323.

COLOMBO, P., C. MARCEÑOy R. PRISCIOTTA -1983- Números cromosómicos de plantas occidentales 200-210. Anal. Jard. Bot. Madrid, 39(2):519-524.

DAHLGREN, R., T. KARLSSON \& P. LARSSEN -1971- Studies on the flora of the Balearic Island. I. Chromosome numbers in Balearic angiosperms. Bot. Not., 124:249-264.

DE LEONARDIS, W., P. PAVONE, M.C TERRASI \& A. ZIZZA -1981- Numeri cromosomici per la Flora Italiana: $814-830$. Inf. Bot. Ital., 13:158-167.

EL-BABA, J. -1976- Contribution à l'étude cytotaxinomique et palynologique des Trifolium de la Méditerranée orientale. Rev. Biol. Ecol. Médit., 3(2):23-40.

ELLERSTRöM, S. \& J. SJODIN -1966- Frequency and vitality of aneuploids in a population of tetraploid red clover. Hereditas, 55:166-182.

EVANS, A.M. -1955- The production and identification of polyploids in red clover, white clover and lucerne. New Phytol., 54:149-162.

EVANS, A.M. -1962- Species hybridization in Trifolium. I. Methods of overcoming species incompatibility. Euphytica, 11(2):164-176.

FERNANDES, A. \& M. QUEIROS -1978Contribution à la connaisance cytotaxinomique des spermatophyta du Portugal IV. Leguminosae (suppl. 3). Bol. Soc. Brot. sér. 2 , 52:79-159.

FERNANDES, A. \& M.F. SANTOS -1971Contribution à la connaisance cytotaxinomique des spermatophyta du Portugal IV. Leguminosae. Bol. Soc. Brot. sér. 2, 45:177226.

FERNANDES, A. \& M.F. SANTOS -1975Contribution à la connaisance cytotaxinomique des spermatophyta du Portugal IV. Leguminosae (suppl. 1). Bol. Soc. Brot. sér. 2 , 49:173-196.

FERNANDES, A., M.F. SANTOS \& M. QUEIROS -1977- Contribution à la connaisance 
cytotaxinomique des spermatophyta du Portugal IV. Leguminosae (suppl. 2). Bol. Soc. Brot. sér. 2, 51:137-186.

GADELLA, T.W.J. \& E. KLIPHUIS -1963Chromosome numbers of flowering plants in the Netherlands. Acta Bot. Neerl., 12:195-230.

GADELLA, T.W.J. \& E. KLIPHUIS -1966Chromosome numbers of flowering plants in the Netherlands. II. Proc. Konink1. Netherl. Akad. Wetensch. ser. c, 70(1):7-20.

GADELLA, T.W.J. \& E. KLIPHUIS - 1968 a- In: A. LÖVE (ed.) IOPB chromosome number reports XVI. Taxon, 17:200-201.

GADELLA, T.W.J. \& E. KLIPHUIS -1968 bChromosome numbers of flowering plants in the Netherlands. IV. Proc. Koninkl. Netherl. Akad. Wetwnsch., ser. c, 71:168-183.

GADELLA, T.W.J.\&E. KLIPHUIS-1972-Studies in chromosome numbers of Yugoslavian angiosperms. Acta Bot. Croat., 31:91-103.

GONZÁLEZ-BERNÁLDEZ,F., A.M. SÁNCHEZ DE RIVERA y M.A. ANGULO -1973- Estudios cromosómicos en el género Trifolium IV. Lagascalia, 3(2):195-203.

HOLUB, J., J. MEESICEK \& V. JAVIURKARA 1972- Anotated Chromosome Counts of Czechoslovak Plants (31-66). Folia Geobot. Phytotax. (Praha), 7:167-202.

HUMPHRIES, C.J., B.G. MURRAY, G. BOCQUET \& K.N. VASUDEVAN -1978Chromosome numbers of phanerogams from Marocco and Algeria. Bot. Not., 131:391-404.

KARPECHENKO, G.D. -1925- Karyologische studien über die Gattung Trifolium L.. Bull. Appl. Bot. Pl.-Breed. (Leningrad.), 14:271279.

KAWAKAMI, I. -1930- Chromosome numbers in Leguminosae. Bot. Mag. Tokyo, 44:319-328.

KLIPHUIS, E. -1962- Chromosome number of some annual Trifolium species, ocurring in the Netherlands. Acta Bot. Neerl., 11;90-92.

KLIPHUIS, E.K. \& J.H. WIEFFERING - 1979- In: A. LÖVE (ed.) IOPB chromosome number reports LXIV. Taxon, 28:398-400.

KODAMA, A. -1967-Cytological studies on root nodules of some species in Leguminosae II. Bot. Mag. Tokyo, 80:92-99.

KODAMA, A. -1970- Cytological and morphological studies on the plant tumors. I. Root nodules of some Leguminosae. Jour. Sci. Hiroshima Univ. ser. B, 2(13):223-260.

KOZUHAROV, S.I., B. KUZMANOZ \& T. MARKOVA - 1972- In: A. LÖVE (ed.) IOPB chromosome number reports XXXVI. Taxon, 21:336-337.

KOZUHAROV, S.I., A.V. PETROVA \& T. MARKOVA -1973- In: A. LÖVE (ed.) IOPB chromosome number reports XL. Taxon, 22:287-288.

KOZUHAROV, S.I., A.V. PETROVA \& T.
MARKOVA -1974- In: A. LÖVE (ed.) IOPB chromosome number reports XLIV. Taxon, 23:373-380.

KOZUHAROV, S.I., A.V. PETROVA \& T MARKOVA -1975- In: A. LÖVE (ed.) IOPB chromosome number reports XLVII. Taxon, 24:145-146.

KROGULEVICH, R.E. -1976- Chromosome numbers of plant species from the Tunkinsky Alpes (East Sayan). News Sib. Dep. Akad. Sci. URRS, ser. Biol., 15(3):46-52.

KUZMANOV, B.A. \& G. STANCEV -1972- In: A. LÖVE (ed.) IOPB chromosome number reports XXXVIII. Taxon, 21:681.

KUZMANOV, B.A. \& G. STANCEV -1973- In: A. LÖVE (ed.) IOPB chromosome number reports XL. Taxon, 22:291.

LABADIE, J. -1979- In: A. LÖVE (ed.) IOPB chromosome number reports LXI. Taxon, 28:628-629.

LARSEN, K. -1956- Chromosome studies in some Mediterranean and South European flowering plants. Bot. Not., 109:293-307.

LARSEN, K. -1960- Cytological and experimental studies on the flowering plants of the Canary Islands. Biol. Meddel. Kong. Danske Vid. Selsk, 11(3):1-60.

LEVAN, A. -1942- Plant breeding induction of polyploidy and some results in clover. Hereditas, 28(1/2):245-246.

LEVAN, A. -1945- Polyploidi foeraedlingens nuvarande laege. Sveriges Utsadesförening Tidsk., 1945:109-143.

LEVAN, A., K. FREDGA \& A.A. SANDBERG 1965- Nomenclature for centromeric position on chromosomes. Hereditas, 52:201-220.

LÖVE, A. \& E. KJELLQVIST -1974Cytotaxonomy of Spanish plants. Dicotyledons Caesalpiniaceae-Asteraceae. Lagascalia, 4:152-211.

LÖVE, A, \& D. LÖVE -1944- Cytotaxonomical studies on boreal plants III. Some new chromosome numbers of Scandinavian plants. Ark. Bot., 31(12):1-22.

LÖVE, A, \& D. LÖVE -1956- Cytotaxonomical conspectus of the Icelanding flora. Acta Horti. Gothob., 20(4):65-291.

LÖVE, A. \& D. LÖVE -1961- Chromosome numbers of Central and Northwest European Plant species. Op. Bot. (Lund.), 5:1-581.

MAJOVSKY, J. PONER TODOS LOS AUTORES -1970 a- Index of chromosome numbers of Slovakian flora. Acta Fac. Rer. Nat. Univ. Comen. Bot., 16:1-26.

MAJOVSKY, J. PONER TODOS LOS AUTORES. - 1970 b- Index of chromosome numbers of Slovakian flora (Part 2). Acta Fac. Rer. Nat. Univ. Comen. Bot., 18:45-60.

MAJOVSKY, J. PONER TODOS LOS AUTORES -1974- Index of chromosome numbers of 
Slovakian flora (Part 3). Acta Fac. Rer. Nat. Univ. Comen. Bot., 22:1-20.

MAJOVSKY, J. PONER TODOS LOS AUTORES - 1975- Index of chromosome numbers of Slovakian flora (Part 4). Acta Fac. Rer. Nat. Univ. Comen. Bot., 23:1-23.

MAJOVSKY, J. PONER TODOS LOS AUTORES . 1978-Index of chromosome numbers of Slovakian flora (Part 6). Acta Fac. Rer. Nat. Univ. Comen. Bot., 26:1-42.

MORIYA, A. \& A. KONDO -1950- Cytological studies of forage plants. II. Legumes. Jap. Jour. Genet., 25:131-134.

MULLIGAN, G.A. -1984- Chromosome numbers of some plant native and naturalized in Canada. Naturaliste Canad., 111:447-449.

MUÑOZ, A. -1992 a- Estudio taxonómico del género Trifolium en la Península Ibérica. Tesis Doctorales en Microfichas $n^{\circ} 20$, Universidad de Sevilla.

MUÑOZ, A. - 1992 b- Trifolium sect. Trifolium en la Península Ibérica. Acta Bot. Malacitana, 17:79-118.

NAPOLI, M. y A. ZIZZA -1984- Números cromosomáticos de plantas occidentales 270279. Anal. Jard. Bot. Madrid, 40(2):451-455.

NATARAJAN, G. -1978- In: A. LÖVE (ed.) IOPB chromosome number reports LXII. Taxon, 27:519-535.

NIELSEN, I. -1975- Chromosome count in the Genus Trifolium. Bot. Tidsskr., 70(2/3):180183.

NILSSON, Q. \& P. LASSEN -1971- Chromosome numbers of vascular plants from Austria, Mallorca and Yugoslavia. Bot. Not., 124:270-276.

NODA, K. -1946- Chromosome studies in clover plants. Jap. Jour. Genet., 21(5/6):93-96.

PARROT, W.A. \& R.R: SMITH - 1984-Production of $2 \mathrm{n}$ pollen in red clover. Crop Sci., 24:469472.

PARROT, W.A., R.R. SMITH \& M.M. SMITH 1985- Bilateral sexual tetraploidization in red clover. Canad. Jour. Genet. Cytol., 27(1):6468.

PETROVA, A.V. \& S.I. KOZUHAROV -1982 aCytotaxonomic Study of Genus Trifolium L. in Bulgaria. I. Fitologiya (Bulgaria), 19:3-23.

PETROVA, A.V. \& S.I. KOZUHAROV -1982 bCytotaxonomic Study of Genus Trifolium L. in Bulgaria. II. Fitologiya (Bulgaria), 20:20-41.

PODLECH, D. \& A. DIETERLE -1969Chromosomenstudien au afghanistanischen pflanzen. Candollea, 24:185-243.

POGAN, E., R. JZMAILOW PONER TODOS LOS AUTORES -1983- Further studies in chromosome numbers of Polish angiosperms. Part XVII. Acta Biol. Cracov. (Bot.), 25:57-77.

POGAN, E., H. WCISLO, R. JZMAILOW, L. PRZYWARA PONER TODOS LOS AUTORES -1982- Further studies in chromosome numbers of Polish angiosperms. Part XVI. Acta Biol. Cracov. (Bot.), 24:159-189.

POVILAITIS, B. \& J.W. BOYES -1956- A cytological study of autotetraploid red clover. Amer. Jour. Bot., 43(3):169-174.

PRITCHARD, A.J. -1967- The somatic chromosomes of Trifolium cherleri L., T.hirtum All., T.ligusticum Balb. and T.scabrum L.. Caryologia, 27(1):17-32.

PRITCHARD, A.J. -1969- Chromosome numbers in some species of Trifolium. Aust. Jour. Agric. Res., 20:883-887.

PUTIYEVSKY, E. \& J. KATZNELSON -1970Chromosome number and genetic system in several Trifolium species related to T.alexandrinum. Chromosoma (Berlin), 30:476-482.

PUTIYEVSKY, E. \& J. KATZNELSON -1972Cytology and crossability of several mediterranean Trifolium species. Israel Jour. Bot., 21:179-181.

REESE, G. -1953- Ergänzende Mitteilungen über die Chromosomenzahler mitteleuropäischer Gefässpflanzen. I. Ber. Deutsch. Bot. Ges., 66(1):66-74

RODRIGUES, J.M.M. -1953-Contribuçao parao conhecimiento cariológica das halófitas e psamófitas literais. Diss. Univ. Coimbra.

ROMERO, C. -1986- A new method for stimating karyotype asimetry. Taxon, 35:526-530.

SAREEN, T.S. \& R. TREHAN -1977- Cytological studies in some Papilionaceae. Proc. Ind. Sci. Congr. Assoc., 64:156.

SCHWER, J.F. \& R.W. CLEVELAND -1972Tetraploid and triploid interspecific hybrids of Trifolium pratense L., T.diffusum Erhr. and some related species. Crop Sci., 12:419-422.

SIKKA, S.M., M.S. SWAMINATHAN \& R.K. MEHTA -1958-Induced polyploidy in Egyptian and Indian clovers. Nature (London), 181:3233.

SMALL, J.G.C. \& A. JOFFE -1967- Physiological studies on the genus Trifolium with special reference to the South African species. I. Germination. S. Afr. Agric. Sci., 10:123-134.

SNOW, R. -1963- Alcoholic hidrochloric acidcarmine as a stain for chromosome in squash preparations. Stain Technol., 38:9-13.

STEBBINS, G.L. -1938- Cytological characteristics associated with the different growth habits in the dicotyledons. Amer. Jour. Bot., 25:189198.

STEBBINS, G.L. -1971- Chromosomal evolution in higher plants. London.

STRID, A. -1971- Chromosome numbers in some Albanian angiosperms. Bot. Not., 124:490-496.

STRID, A. -1980- In: A. LÖVE (ed.) IOPB chromosome number reports LXIX. Taxon, 29:709-710

TATUNO, S. \& A. KODAMA -1965- Cytological 
studies on root nodules of some species in Leguminosae. I. Bot. Mag. Tokyo, 78:503-509.

TAYLOR, N.L., M.K. ANDERSON, K.H. QUESENBERRY \& L. WATSON -1976Doubling the chromosome number of Trifolium species using nitrous oxide. Crop Sci., 16:516518.

TAYLOR, N.L., R.F. QUARTES \& M.K. ANDERSON -1980- Methods of overcoming interspecific barriers in Trifolium. Euphytica, 29:441-450.

TAYLOR, N.L., W.H. STRAUBE, G.B. COLLINS \& W.A. KENDALL -1963- Interspecific hybridization of red clover (Trifolium pratense L.- Crop Sci., 3:549-552.

TISCHLER, G. -1934- Die Beden tungen der Polyploidie für die Verbreitung der Angiospermen, erläntert an den Arten Schlesswig-Holsteins mit Ausblicken auf audere Florengebiete. Bot. Jahrb., 67:1-36.

TISCHLER, G. -1937- Die Halligenflora der Nordsee in Lichte cytologischer Forchung. Cytologia, Fuji Jub. Vol., 162-170.

TJIO, J.H. \& A. LEVAN -1950- The use of oxyquinoleine in chromosome analysis. Anal. Est. Exp. Aula Dei, 2:21-64.

TOFTE, J.E. \& R.R SMITH -1989- Cytology of bilaterally derived tetraploid red clover. Crop Sci., 29:37-41.

TURESSON, G: -1962- Results of colchicine doubling in the red, alsike and white clover. Agr. Hort. Genet., 20:111-135.

UOTILA, P. \& K. PELLINEN - 1985-Chromosome numbers in vascular plants from Finland. Acta Bot. Fenn., 1985:130.

VALDÉS, B. y F. GONZÁLEZ-BERNÁLDEZ 1972- Trifolium baeticum Boiss. y Trifolium pallidum Waldst. \& Kit.. Lagascalia, 2(2):189191.

VAN LOON, J.C. -1974- A cytological investigation of flowering plants from the Canary Islands. Acta Bot. Neerl., 23(2):113124.

VAN LOON, J.C. -1980- In; A. LÖVE (ed.) IOPB chromosome number reports LXIV. Taxon, 29:718-720.

VAN LOON, J.C. \& H. DE JONG -1978- In: A. LÖVE (ed.) IOPB chromosome number reports LIX. Taxon, 27:53-61.

VANLOON, J.C. \& B. KIEFT - 1980- In: A. LÖVE (ed.) IOPB chromosome number reports LXVIII. Taxon, 29:538-542.

VAN LOON, J.C. \& A.K. VAN SETTEN -1982In: A. LÖVE (ed.) IOPB chromosome number reports LXXVI. Taxon, 31:589-592.

VAN LOON, J.C., T.W.J. GADELLA \& E. KLIPHUIS -1971-Cytological studies in some flowering plants from Southern France. Acta Bot. Neerl., 20:157-166.

WEXELSEN, H. -1928- Chromosome numbers and morphology in Trifolium. Univ. Calif. Publ. Agr. Sci., 2(13):355-374.

WEXELSEN, H. \& K. MICKAELSEN -1958- The effect of neutron treatment of seed and seedling of diploid and tetraploid red clover (Trifolium pratense). Physiol. Pl., 3:374-381.

WIPF, L. -1939- Chromosome numbers in root nodules and root tips of certain Leguminosae. Bot. Gaz., 101:51-67.

WIPF, L. \& D.C. COOPER -1938- Chromosome numbers in nodules and roots of red clover, common vetch, and garden pea. Proc. Nat. Acad. Sci. U.S., 24(2):87-91.

WULFF, H.D. -1939- Chromosomenstudium an der Schleswigholsteinischen AngiospermenFlora IV. Ber. Deutsch. Bot. Ges., 57:424-431.

ZÄBORSKY, J. -1971 - Trifolium diffusum Ehrh., neue Art der Flora der Slowakie und Bermerkungen zu deren Karyologie. Acta Fac. Rer. Nat. Univ. Comen. Bot., 19:237-248.

ZOHARY, M. -1971- A Revision of the Species of Trifolium Sect. Trifolium (Leguminosae). I. Introduction. Candollea, 26:297-308.

ZOHARY, M. \& D. HELLER -1984- The Genus Trifolium. Jerusalem.

Aceptado para su publicación en Marzo de 1993

Dirección del autor: Dpto. de Biología y Producción de los Vegetales, Escuela Universitaria de Ingeniería Técnica Agrícola, Universidad de Extremadura. 06071 Badajoz, España. 